Supplement of Atmos. Chem. Phys., 18, 9475-9497, 2018

https://doi.org/10.5194/acp-18-9475-2018-supplement

(C) Author(s) 2018. This work is distributed under

the Creative Commons Attribution 3.0 License.

(c) (1)

Supplement of

\title{
Simulating $\mathrm{CH}_{4}$ and $\mathrm{CO}_{2}$ over South and East Asia using the zoomed chemistry transport model LMDz-INCA
}

Xin Lin et al.

Correspondence to: Xin Lin (xin.lin@1sce.ipsl.fr)

The copyright of individual parts of the supplement might differ from the CC BY 3.0 License. 


\section{Supplementary materials}

2 Table S1 The mean bias ( \pm s.d.) and RMSE of the simulated annual gradient for different 3 station groups: (a) $\mathrm{CH}_{4}$ (unit: ppb), (b) $\mathrm{CO}_{2}$ (ppm). Results from both ZAs and STs are 4 presented. Statistics are given for stations outside and within the zoomed region, as well as 5 for stations of different types within the zoomed region.

$6 \quad(\mathbf{a})$

\begin{tabular}{|l|l|r|r|r|r|r|r|}
\hline model version & statistics & outside Z & within Z & marine & mountain & coastal & continental \\
\hline \multirow{2}{*}{ ST19_ED42 } & MB & $-6.4 \pm 4.8$ & $0.0 \pm 21.0$ & $6.7 \pm 23.5$ & $-3.2 \pm 10.2$ & $-7.8 \pm 22.3$ & $2.3 \pm 35.7$ \\
\cline { 2 - 8 } & RMSE & 7.9 & 20.4 & 22.0 & 9.6 & 19.8 & 29.2 \\
\hline \multirow{2}{*}{ ZA19_ED42 } & MB & $-0.6 \pm 8.0$ & $3.8 \pm 16.5$ & $15.0 \pm 18.0$ & $-1.8 \pm 7.9$ & $8.4 \pm 13.4$ & $-10.4 \pm 19.3$ \\
\cline { 2 - 8 } & RMSE & 7.7 & 16.4 & 22.0 & 7.2 & 13.8 & 18.9 \\
\hline \multirow{2}{*}{ ST39_ED42 } & MB & $-6.8 \pm 4.4$ & $0.1 \pm 22.3$ & $7.1 \pm 24.6$ & $-5.2 \pm 10.7$ & $-7.3 \pm 25.5$ & $4.9 \pm 36.6$ \\
\cline { 2 - 8 } & RMSE & 8.0 & 21.6 & 23.1 & 10.9 & 22.1 & 30.3 \\
\hline \multirow{2}{*}{ ZA39_ED42 } & MB & $-1.2 \pm 7.9$ & $6.4 \pm 17.5$ & $17.6 \pm 17.8$ & $-1.9 \pm 7.8$ & $11.9 \pm 14.5$ & $-4.0 \pm 25.0$ \\
\cline { 2 - 8 } & RMSE & 7.7 & 18.1 & 23.7 & 7.2 & 16.8 & 20.8 \\
\hline
\end{tabular}

$7 \quad(\mathbf{b})$

\begin{tabular}{|c|l|r|r|r|r|r|r|}
\hline $\begin{array}{c}\text { model } \\
\text { version }\end{array}$ & statistics & outside Z & within Z & marine & mountain & coastal & continental \\
\hline \multirow{2}{*}{ ST19_ED42 } & MB & $-0.6 \pm 0.5$ & $-0.1 \pm 2.5$ & $-0.3 \pm 1.7$ & $0.5 \pm 4.0$ & $-1.5 \pm 1.8$ & $0.2 \pm 1.9$ \\
\cline { 2 - 8 } & RMSE & 0.8 & 2.4 & 1.5 & 3.6 & 2.1 & 1.7 \\
\hline \multirow{2}{*}{ ZA19_ED42 } & MB & $-0.9 \pm 1.4$ & $0.0 \pm 2.5$ & $-0.3 \pm 2.2$ & $0.2 \pm 3.9$ & $-0.1 \pm 3.0$ & $0.0 \pm 1.8$ \\
\cline { 2 - 8 } & RMSE & 1.7 & 2.5 & 1.9 & 3.5 & 2.4 & 1.6 \\
\hline \multirow{2}{*}{ ST39_ED42 } & MB & $-0.4 \pm 0.7$ & $0.0 \pm 2.5$ & $-0.1 \pm 1.7$ & $0.5 \pm 4.0$ & $-1.2 \pm 1.9$ & $0.3 \pm 2.1$ \\
\cline { 2 - 8 } & RMSE & 0.8 & 2.5 & 1.5 & 3.6 & 2.0 & 1.9 \\
\hline \multirow{2}{*}{ ZA39_ED42 } & MB & $-0.9 \pm 1.4$ & $0.1 \pm 2.6$ & $-0.2 \pm 2.1$ & $0.2 \pm 3.8$ & $0.3 \pm 3.3$ & $0.1 \pm 2.0$ \\
\cline { 2 - 8 } & RMSE & 1.6 & 2.5 & 1.9 & 3.4 & 2.7 & 1.8 \\
\hline
\end{tabular}


9 Table S2 The observed and simulated mean annual gradient of (a) $\mathrm{CH}_{4}$ and (b) $\mathrm{CO}_{2}$ at 10 stations mentioned in Section 3.1.1 and Section 3.1.2. The bias reduction rates (in percentage)

11 of ZAs compared to STs are also given for both 19-layer and 39-layer simulations.

a)

\begin{tabular}{lccccccc}
\hline CH4 & $\begin{array}{c}\text { OBS } \\
(\mathbf{p p b})\end{array}$ & $\begin{array}{c}\text { ST19 } \\
(\mathbf{p p b})\end{array}$ & $\begin{array}{c}\text { ZA19 } \\
(\mathbf{p p b})\end{array}$ & $\begin{array}{c}\text { Bias } \\
\text { reduction }\end{array}$ & $\begin{array}{c}\text { ST39 } \\
(\mathbf{p p b})\end{array}$ & $\begin{array}{c}\text { ZA39 } \\
(\mathbf{p p b})\end{array}$ & $\begin{array}{c}\text { Bias } \\
\text { reduction }\end{array}$ \\
\hline PON & $32.4 \pm 12.4$ & $2.5 \pm 11.6$ & $31.1 \pm 7.7$ & $95.6 \%$ & $0.4 \pm 11.9$ & $34.1 \pm 7.8$ & $94.7 \%$ \\
SDZ & $90.0 \pm 15.4$ & $125.1 \pm 18.8$ & $86.8 \pm 16.0$ & $91.0 \%$ & $128.5 \pm 19.3$ & $100.4 \pm 22.4$ & $73.0 \%$ \\
TAP & $64.9 \pm 10.7$ & $79.5 \pm 8.1$ & $88.6 \pm 8.4$ & n.a. & $83.9 \pm 7.5$ & $93.3 \pm 7.8$ & n.a. \\
UUM & $38.6 \pm 5.6$ & $46.1 \pm 9.7$ & $42.8 \pm 13.3$ & $44.1 \%$ & $49.0 \pm 11.6$ & $49.1 \pm 8.9$ & n.a. \\
\hline
\end{tabular}

b)

\begin{tabular}{cccccccc}
\hline CO2 & $\begin{array}{c}\text { OBS } \\
(\mathbf{p p m})\end{array}$ & $\begin{array}{c}\text { ST19 } \\
(\mathbf{p p m})\end{array}$ & $\begin{array}{c}\text { ZA19 } \\
(\mathbf{p p m})\end{array}$ & $\begin{array}{c}\text { Bias } \\
\text { reduction }\end{array}$ & $\begin{array}{c}\text { ST39 } \\
(\mathbf{p p m})\end{array}$ & $\begin{array}{c}\text { ZA39 } \\
(\mathbf{p p m})\end{array}$ & $\begin{array}{c}\text { Bias } \\
\text { reduction }\end{array}$ \\
\hline $\mathrm{PON}$ & $2.7 \pm 1.6$ & $1.3 \pm 0.3$ & $1.8 \pm 0.5$ & $35.2 \%$ & $1.5 \pm 0.3$ & $1.9 \pm 0.5$ & $37.0 \%$ \\
$\mathrm{SDZ}$ & $6.8 \pm 0.5$ & $8.8 \pm 1.3$ & $7.7 \pm 1.9$ & $57.9 \%$ & $9.3 \pm 1.5$ & $8.1 \pm 2.3$ & $48.1 \%$ \\
$\mathrm{TAP}$ & $6.9 \pm 1.8$ & $7.2 \pm 0.8$ & $10.2 \pm 0.8$ & n.a. & $7.5 \pm 1.0$ & $10.8 \pm 1.1$ & n.a. \\
\hline
\end{tabular}


13 Table S3 The correlation coefficients between the simulated and observed synoptic 14 variability of $\mathrm{CH}_{4}$ (a) and $\mathrm{CO}_{2}$ (b) at PON over the period 2006-2013. The synoptic 15 variability is calculated from residuals from the smoothed fitting curve.

$16 \quad$ (a) $\mathrm{CH}_{4}$

\begin{tabular}{lccccc}
\hline \multicolumn{1}{c}{ Months } & N. of Samples & ST19_ED42 & ZA19_ED42 & ST39_ED42 & ZA39_ED42 \\
\hline Jan.-Mar. & 132 & $0.40^{* * *}$ & $0.39^{* * * *}$ & $0.42^{* * * *}$ & $0.42^{* * * *}$ \\
Apr.-Jun. & 81 & $0.46^{* * *}$ & $0.43^{* * *}$ & $0.49^{* * *}$ & $0.43^{* * *}$ \\
Jul.-Sep. & 123 & $0.48^{* * *}$ & $0.46^{* * *}$ & $0.48^{* * *}$ & $0.45^{* * *}$ \\
Oct.-Dec. & 88 & $0.36^{* * *}$ & $0.49^{* * *}$ & $0.39^{* * *}$ & $0.53^{* * *}$ \\
All & 424 & $0.40^{* * *}$ & $0.45^{* * *}$ & $0.42^{* * *}$ & $0.47^{* * *}$ \\
\hline
\end{tabular}

17

(b) $\mathrm{CO}_{2}$

\begin{tabular}{lccccc}
\hline \multicolumn{1}{c}{ Months } & N. of Samples & ST19_ED42 & ZA19_ED42 & ST39_ED42 & ZA39_ED42 \\
\hline Jan.-Mar. & 124 & -0.10 & $-0.24^{* *}$ & -0.08 & $-0.20^{*}$ \\
Apr.-Jun. & 69 & -0.20 & -0.23 & -0.21 & $-0.24^{*}$ \\
Jul.-Sep. & 105 & $-0.20^{*}$ & 0.05 & $-0.22^{*}$ & 0.02 \\
Oct.-Dec. & 83 & 0.05 & 0.08 & 0.02 & 0.06 \\
All & 381 & $-0.11^{*}$ & $-0.11^{*}$ & $-0.11^{*}$ & $-0.11^{*}$ \\
\hline
\end{tabular}

18 

(a) GSN

Table S4 The statistics between the simulated and observed mean diurnal cycles of $\mathrm{CH}_{4}$ for three exemplified stations GSN (a), PON (b) and BKT (c) over specific study periods. For BKT, results from outputs extracted at a lower model level (Level=2) are presented in (d). For each station, correlation coefficients and ratios of amplitudes are calculated from the simulated and observed diurnal cycles averaged over all the sampling days in a month with a complete 24-hour profile.

\begin{tabular}{cccccccccccc}
\hline \multirow{2}{*}{ Month } & N. of & Amplitudes & \multicolumn{2}{c}{ ST19_ED42 } & \multicolumn{2}{c}{ ZA19_ED42 } & \multicolumn{2}{c}{ ST39_ED42 } & \multicolumn{2}{c}{ ZA39_ED42 } \\
\cline { 4 - 11 } & Days & $(\mathrm{ppb})$ & $\mathrm{R}$ & $\mathrm{A}_{\mathrm{m}} / \mathrm{A}_{\mathrm{o}}$ & $\mathrm{R}$ & $\mathrm{A}_{\mathrm{m}} / \mathrm{A}_{\mathrm{o}}$ & $\mathrm{R}$ & $\mathrm{A}_{\mathrm{m}} / \mathrm{A}_{\mathrm{o}}$ & $\mathrm{R}$ & $\mathrm{A}_{\mathrm{m}} / \mathrm{A}_{\mathrm{o}}$ \\
\hline 200801 & 16 & 13.2 & 0.33 & 0.41 & 0.39 & 1.15 & 0.29 & 0.42 & 0.40 & 1.39 \\
200802 & 16 & 13.8 & $0.54^{* *}$ & 1.50 & $0.72^{* * *}$ & 0.95 & $0.49^{*}$ & 1.56 & $0.75^{* * *}$ & 0.93 \\
200803 & 24 & 17.6 & $0.48^{*}$ & 0.11 & $0.51^{*}$ & 0.66 & $0.50^{*}$ & 0.14 & $0.45^{*}$ & 0.75 \\
200804 & 13 & 27.1 & $0.64^{* * *}$ & 0.47 & $0.80^{* * *}$ & 0.75 & $0.69^{* * *}$ & 0.35 & $0.78^{* * *}$ & 0.81 \\
200805 & 15 & 28.4 & $-0.81^{* * * *}$ & 0.24 & $0.70^{* * *}$ & 0.52 & $-0.86^{* * *}$ & 0.18 & $0.63^{* *}$ & 0.43 \\
200806 & 19 & 45.6 & $0.78^{* * *}$ & 0.17 & $0.79^{* * *}$ & 0.56 & $0.78^{* * *}$ & 0.20 & $0.73^{* * *}$ & 0.58 \\
200807 & 12 & 24.5 & -0.05 & 0.75 & 0.00 & 0.43 & -0.11 & 0.85 & 0.32 & 0.44 \\
200808 & 14 & 58.4 & $0.83^{* * * *}$ & 0.25 & $0.67^{* * *}$ & 0.72 & $0.86^{* * *}$ & 0.28 & $0.76^{* * *}$ & 0.74 \\
200809 & 1 & 63.5 & $0.64^{* *}$ & 0.57 & 0.08 & 0.35 & $0.59^{* *}$ & 0.73 & 0.40 & 0.46 \\
200810 & 12 & 28.2 & $0.48^{*}$ & 0.37 & -0.29 & 0.85 & $0.47^{*}$ & 0.45 & -0.35 & 0.80 \\
200811 & 12 & 19.3 & -0.30 & 0.69 & 0.28 & 0.86 & $-0.52^{* *}$ & 1.08 & -0.09 & 0.74 \\
200812 & 17 & 17.0 & 0.09 & 0.53 & 0.35 & 0.99 & $0.43^{*}$ & 0.65 & $0.48^{*}$ & 1.03 \\
\hline
\end{tabular}

\section{(b) PON}

\begin{tabular}{ccccccccccc}
\hline \multirow{2}{*}{ Month } & \multirow{2}{*}{ N. of } & Amplitudes & \multicolumn{2}{c}{ ST19_ED42 } & \multicolumn{2}{c}{ ZA19_ED42 } & \multicolumn{2}{c}{ ST39_ED42 } & \multicolumn{2}{c}{ ZA39_ED42 } \\
\cline { 5 - 10 } & Days & (ppb) & $\mathrm{R}$ & $\mathrm{A}_{\mathrm{m}} / \mathrm{A}_{\mathrm{o}}$ & $\mathrm{R}$ & $\mathrm{A}_{\mathrm{m}} / \mathrm{A}_{\mathrm{o}}$ & $\mathrm{R}$ & $\mathrm{A}_{\mathrm{m}} / \mathrm{A}_{\mathrm{o}}$ & $\mathrm{R}$ & $\mathrm{A}_{\mathrm{m}} / \mathrm{A}_{\mathrm{o}}$ \\
\hline 201108 & 4 & 187.4 & $0.56^{* * *}$ & 0.04 & $0.79^{* * *}$ & 0.15 & $0.71^{* * * *}$ & 0.06 & $0.76^{* * * *}$ & 0.15 \\
201109 & 14 & 163.1 & $0.96^{* * *}$ & 0.08 & $0.91^{* * *}$ & 0.40 & $0.93^{* * *}$ & 0.09 & $0.84^{* * * *}$ & 0.37 \\
201210 & 25 & 133.5 & $0.87^{* * *}$ & 0.13 & $0.96^{* * *}$ & 0.50 & $0.83^{* * *}$ & 0.18 & $0.95^{* * *}$ & 0.68 \\
201211 & 26 & 229.6 & $0.95^{* * *}$ & 0.12 & $0.97^{* * *}$ & 0.33 & $0.95^{* * *}$ & 0.16 & $0.98^{* * *}$ & 0.40 \\
201212 & 28 & 206.6 & $0.88^{* * *}$ & 0.06 & $0.98^{* * *}$ & 0.31 & $0.96^{* * *}$ & 0.07 & $1.00^{* * *}$ & 0.34 \\
201301 & 27 & 309.0 & $0.89^{* * *}$ & 0.05 & $0.98^{* * *}$ & 0.22 & $0.94^{* * *}$ & 0.05 & $0.98^{* * *}$ & 0.26 \\
201302 & 20 & 238.9 & $0.79^{* * *}$ & 0.08 & $0.97^{* * *}$ & 0.27 & $0.85^{* * *}$ & 0.08 & $0.97^{* * *}$ & 0.32 \\
201303 & 29 & 146.9 & $0.85^{* * *}$ & 0.10 & $0.96^{* * *}$ & 0.48 & $0.91^{* * *}$ & 0.10 & $0.95^{* * *}$ & 0.60 \\
201304 & 25 & 121.6 & $0.76^{* * *}$ & 0.09 & $0.94^{* * *}$ & 0.40 & $0.83^{* * *}$ & 0.10 & $0.90^{* * *}$ & 0.42 \\
201305 & 15 & 78.9 & $0.93^{* * *}$ & 0.15 & $0.92^{* * *}$ & 0.41 & $0.90^{* * *}$ & 0.13 & $0.93^{* * *}$ & 0.36 \\
\hline
\end{tabular}

${ }^{*} p<0.05 ;{ }^{* *} p<0.01 ;{ }^{* * *} p<0.001$

(c) BKT, at the station level

\begin{tabular}{cccccccccccc}
\hline \multirow{2}{*}{ Month } & \multirow{2}{*}{ N. of } & Amplitudes & \multicolumn{2}{c}{ ST19_ED42 } & \multicolumn{2}{c}{ ZA19_ED42 } & \multicolumn{2}{c}{ ST39_ED42 } & \multicolumn{2}{c}{ ZA39_ED42 } \\
\cline { 4 - 11 } & Days & $(\mathrm{ppb})$ & $\mathrm{R}$ & $\mathrm{A}_{\mathrm{m}} / \mathrm{A}_{\mathrm{o}}$ & $\mathrm{R}$ & $\mathrm{A}_{\mathrm{m}} / \mathrm{A}_{\mathrm{o}}$ & $\mathrm{R}$ & $\mathrm{A}_{\mathrm{m}} / \mathrm{A}_{\mathrm{o}}$ & $\mathrm{R}$ & $\mathrm{A}_{\mathrm{m}} / \mathrm{A}_{\mathrm{o}}$ \\
\hline 201301 & 27 & 67.4 & -0.09 & 0.19 & $0.80^{* * *}$ & 0.24 & $-0.49^{*}$ & 0.12 & $0.89^{* * *}$ & 0.34 \\
201302 & 17 & 32.5 & -0.12 & 0.33 & 0.08 & 0.28 & -0.18 & 0.28 & $0.47^{*}$ & 0.26 \\
201303 & 23 & 83.6 & -0.22 & 0.14 & $0.70^{* * *}$ & 0.21 & $-0.61^{* *}$ & 0.10 & $0.81^{* * *}$ & 0.26 \\
201304 & 20 & 47.9 & -0.19 & 0.21 & -0.05 & 0.21 & -0.28 & 0.19 & -0.02 & 0.21
\end{tabular}




\begin{tabular}{ccccccccccc}
201305 & 17 & 33.7 & $-0.60^{* *}$ & 0.31 & $-0.53^{* *}$ & 0.26 & $-0.62^{* *}$ & 0.31 & $-0.42^{*}$ & 0.31 \\
201306 & 18 & 30.6 & 0.18 & 0.92 & 0.17 & 0.51 & 0.03 & 0.84 & $0.43^{*}$ & 0.51 \\
201307 & 19 & 31.1 & -0.24 & 0.46 & -0.21 & 0.27 & -0.37 & 0.47 & -0.13 & 0.32 \\
201308 & 21 & 50.2 & $-0.83^{* * *}$ & 0.14 & $-0.42^{*}$ & 0.13 & $-0.84^{* * *}$ & 0.13 & -0.32 & 0.12 \\
201309 & 8 & 22.6 & $0.51^{*}$ & 0.58 & 0.40 & 0.46 & $0.61^{* *}$ & 0.57 & 0.34 & 0.52 \\
201310 & 16 & 90.8 & -0.27 & 0.09 & 0.20 & 0.07 & $-0.74^{* * *}$ & 0.07 & 0.27 & 0.09 \\
201311 & 21 & 44.7 & -0.11 & 0.24 & -0.07 & 0.24 & -0.12 & 0.19 & -0.24 & 0.22 \\
201312 & 22 & 58.3 & $0.50^{*}$ & 0.17 & $0.87^{* * *}$ & 0.34 & $0.57^{* *}$ & 0.11 & $0.80^{* * *}$ & 0.43 \\
\hline
\end{tabular}

$30 \quad{ }^{*} p<0.05 ;{ }^{* *} p<0.01 ;{ }^{* * *} p<0.001$

31 (d) BKT, at a lower model level (Level=2)

\begin{tabular}{ccccccccccc}
\hline \multirow{2}{*}{ Month } & N. of & Amplitudes & \multicolumn{2}{c}{ ST19_ED42 } & \multicolumn{2}{c}{ ZA19_ED42 } & \multicolumn{2}{c}{ ST39_ED42 } & \multicolumn{2}{c}{ ZA39_ED42 } \\
\cline { 5 - 10 } & Days & $(\mathrm{ppb})$ & $\mathrm{R}$ & $\mathrm{A}_{\mathrm{m}} / \mathrm{A}_{\mathrm{o}}$ & $\mathrm{R}$ & $\mathrm{A}_{\mathrm{m}} / \mathrm{A}_{\mathrm{o}}$ & $\mathrm{R}$ & $\mathrm{A}_{\mathrm{m}} / \mathrm{A}_{\mathrm{o}}$ & $\mathrm{R}$ & $\mathrm{A}_{\mathrm{m}} / \mathrm{A}_{\mathrm{o}}$ \\
\hline 201301 & 27 & 67.4 & $0.78^{* * *}$ & 0.51 & $0.94^{* * *}$ & 0.55 & $0.97^{* * *}$ & 0.87 & $0.98^{* * *}$ & 0.83 \\
201302 & 17 & 32.5 & $0.78^{* * *}$ & 0.99 & $0.87^{* * *}$ & 0.71 & $0.83^{* * *}$ & 1.64 & $0.74^{* * *}$ & 1.21 \\
201303 & 23 & 83.6 & $0.86^{* * *}$ & 0.38 & $0.91^{* * *}$ & 0.50 & $0.89^{* * *}$ & 0.67 & $0.84^{* * *}$ & 0.67 \\
201304 & 20 & 47.9 & $0.72^{* * *}$ & 0.32 & $0.60^{* *}$ & 0.22 & $0.72^{* * *}$ & 0.55 & $0.72^{* * *}$ & 0.38 \\
201305 & 17 & 33.7 & 0.37 & 0.42 & 0.01 & 0.30 & $0.85^{* * *}$ & 0.64 & $0.88^{* * * *}$ & 0.43 \\
201306 & 18 & 30.6 & $0.56^{* *}$ & 1.64 & $0.57^{* *}$ & 0.84 & $0.55^{* *}$ & 2.18 & $0.53^{* *}$ & 1.17 \\
201307 & 19 & 31.1 & $0.72^{* * *}$ & 0.73 & $0.46^{*}$ & 0.30 & $0.82^{* * *}$ & 1.13 & $0.70^{* * *}$ & 0.56 \\
201308 & 21 & 50.2 & $0.65^{* *}$ & 0.24 & $0.53^{* *}$ & 0.16 & $0.83^{* * *}$ & 0.46 & $0.77^{* * *}$ & 0.38 \\
201309 & 8 & 22.6 & 0.10 & 0.80 & 0.09 & 0.63 & -0.22 & 1.24 & -0.13 & 1.26 \\
201310 & 16 & 90.8 & $0.81^{* * *}$ & 0.22 & $0.82^{* * *}$ & 0.13 & $0.95^{* * *}$ & 0.35 & $0.91^{* * *}$ & 0.27 \\
201311 & 21 & 44.7 & $0.44^{* *}$ & 0.43 & 0.38 & 0.29 & $0.70^{* * *}$ & 0.61 & $0.60^{* *}$ & 0.29 \\
201312 & 22 & 58.3 & $0.69^{* * *}$ & 0.55 & $0.81^{* * *}$ & 0.67 & $0.85^{* * *}$ & 1.07 & $0.87^{* * *}$ & 1.02 \\
\hline
\end{tabular}

${ }^{*} p<0.05 ;{ }^{* *} p<0.01 ;{ }^{* * *} p<0.001$ 
(a) GSN

Table S5 The statistics between the simulated and observed mean diurnal cycles of $\mathrm{CO}_{2}$ for three exemplified stations GSN (a), PON (b) and BKT (c) over specific study periods. For BKT, results from outputs extracted at the surface model level (Level=1) are presented in (d). For each station, correlation coefficients and ratios of amplitudes are calculated from the simulated and observed diurnal cycles averaged over all the sampling days in a month with a complete 24-hour profile.

\begin{tabular}{|c|c|c|c|c|c|c|c|c|c|c|}
\hline \multirow{2}{*}{ Month } & \multirow{2}{*}{$\begin{array}{l}\text { N. of } \\
\text { Days }\end{array}$} & \multirow{2}{*}{$\begin{array}{l}\text { Amplitudes } \\
(\mathrm{ppm})\end{array}$} & \multicolumn{2}{|c|}{ ST19_ED42 } & \multicolumn{2}{|c|}{ ZA19_ED42 } & \multicolumn{2}{|c|}{ ST39_ED42 } & \multicolumn{2}{|c|}{ ZA39_ED42 } \\
\hline & & & $\mathrm{R}$ & $\mathrm{A}_{\mathrm{m}} / \mathrm{A}_{\mathrm{o}}$ & $\mathrm{R}$ & $\mathrm{A}_{\mathrm{m}} / \mathrm{A}_{\mathrm{o}}$ & $\mathrm{R}$ & $\mathrm{A}_{\mathrm{m}} / \mathrm{A}_{\mathrm{o}}$ & $\mathrm{R}$ & $\mathrm{A}_{\mathrm{m}} / \mathrm{A}_{\mathrm{o}}$ \\
\hline 200801 & 10 & 2.3 & 0.01 & 0.17 & 0.00 & 0.30 & 0.12 & 0.26 & 0.04 & 0.45 \\
\hline 200802 & 13 & 4.0 & $0.59^{* * *}$ & 0.45 & $0.78^{* * *}$ & 0.27 & $0.56^{* *}$ & 0.47 & $0.81^{* * * *}$ & 0.26 \\
\hline 200803 & 20 & 2.2 & $-0.52^{* *}$ & 0.22 & $0.70^{* * * *}$ & 0.49 & $-0.64^{* *}$ & 0.19 & $0.53^{* *}$ & 0.42 \\
\hline 200804 & 17 & 3.2 & $0.79^{* * * *}$ & 0.27 & $0.74^{* * * *}$ & 0.32 & $0.82^{* * *}$ & 0.27 & $0.72^{* * * *}$ & 0.37 \\
\hline 200805 & 13 & 2.8 & $0.47^{*}$ & 0.12 & -0.36 & 0.14 & 0.33 & 0.03 & $-0.43^{*}$ & 0.12 \\
\hline 200806 & 12 & 3.5 & -0.30 & 0.08 & 0.10 & 0.07 & $-0.42^{*}$ & 0.11 & -0.26 & 0.08 \\
\hline 200807 & 12 & 4.4 & 0.40 & 0.34 & $0.50^{*}$ & 0.18 & $0.54^{* *}$ & 0.33 & $0.70^{* * * *}$ & 0.21 \\
\hline 200808 & 15 & 5.3 & 0.25 & 0.11 & $0.59^{* *}$ & 0.40 & 0.30 & 0.14 & $0.66^{* * * *}$ & 0.36 \\
\hline 200809 & 9 & 5.8 & $-0.73^{\text {**** }}$ & 0.16 & 0.00 & 0.30 & $-0.82^{* * *}$ & 0.15 & -0.26 & 0.33 \\
\hline 200810 & 13 & 4.0 & -0.36 & 0.22 & $-0.74^{* * * *}$ & 0.51 & -0.34 & 0.27 & $-0.74^{* * *}$ & 0.52 \\
\hline 200811 & 8 & 1.8 & $0.90^{* * * *}$ & 0.55 & $0.90^{* * * *}$ & 1.09 & $0.83^{* * *}$ & 0.23 & $0.85^{* * * *}$ & 0.84 \\
\hline 200812 & 20 & 1.7 & $-0.48^{* *}$ & 0.35 & 0.09 & 0.80 & $-0.50^{* *}$ & 0.35 & 0.25 & 0.65 \\
\hline
\end{tabular}

\section{(b) PON}

\begin{tabular}{|c|c|c|c|c|c|c|c|c|c|c|}
\hline \multirow{2}{*}{ Month } & \multirow{2}{*}{$\begin{array}{l}\text { N. of } \\
\text { Days }\end{array}$} & \multirow{2}{*}{$\begin{array}{c}\text { Amplitudes } \\
\text { (ppm) }\end{array}$} & \multicolumn{2}{|c|}{ ST19_ED42 } & \multicolumn{2}{|c|}{ ZA19_ED42 } & \multicolumn{2}{|c|}{ ST39_ED42 } & \multicolumn{2}{|c|}{ ZA39_ED42 } \\
\hline & & & $\mathrm{R}$ & $\mathrm{A}_{\mathrm{m}} / \mathrm{A}_{\mathrm{o}}$ & $\mathrm{R}$ & $\mathrm{A}_{\mathrm{m}} / \mathrm{A}_{\mathrm{o}}$ & $\mathrm{R}$ & $\mathrm{A}_{\mathrm{m}} / \mathrm{A}_{\mathrm{o}}$ & $\mathrm{R}$ & $\mathrm{A}_{\mathrm{m}} / \mathrm{A}_{\mathrm{o}}$ \\
\hline 201108 & 4 & 42.4 & 0.36 & 0.01 & $0.43^{*}$ & 0.03 & $0.41^{*}$ & 0.02 & 0.28 & 0.03 \\
\hline 201109 & 14 & 3 & $0.90^{* * *}$ & 0.02 & $0.83^{* * * *}$ & 0.10 & $0.86^{* * *}$ & 0.02 & $0.72^{* * * *}$ & 0.11 \\
\hline 201210 & 25 & 232 & $0.83^{* * *}$ & 0.04 & $0.94^{* * *}$ & 0.12 & $0.78^{* * * *}$ & 0.06 & $0.95^{* * *}$ & 0.16 \\
\hline 201211 & 26 & 3 & $0.86^{* * *}$ & 0.03 & $0.92^{* * * *}$ & 0.05 & $0.85^{* * *}$ & 0.04 & $0.91^{* * * *}$ & 0.06 \\
\hline 201212 & 28 & 3 & $0.60^{* *}$ & 0.01 & $0.96^{* * *}$ & 0.04 & $0.77^{* * * *}$ & 0.01 & $0.95^{* * *}$ & 0.05 \\
\hline 201301 & 27 & 38.2 & $0.81^{* * *}$ & 0.01 & 0.07 & 0.02 & $0.84^{* * *}$ & 0.01 & 0.02 & 0.02 \\
\hline 201302 & 20 & 36.5 & $0.84^{* * *}$ & 0.02 & 0.34 & 0.02 & $0.81^{* * *}$ & 0.02 & $0.54^{* *}$ & 0.02 \\
\hline 201303 & 29 & 29.9 & $0.88^{* * *}$ & 0.05 & $0.95^{* * *}$ & 0.13 & $0.91^{* * * *}$ & 0.04 & $0.97^{* * * *}$ & 0.17 \\
\hline 201304 & 25 & 19.4 & $0.77^{* * * *}$ & 0.10 & $0.94^{* * * *}$ & 0.35 & $0.80^{* * * *}$ & 0.11 & $0.92^{* * * *}$ & 0.37 \\
\hline 201305 & 15 & 13.4 & $0.89^{* * *}$ & 0.11 & $0.56^{* *}$ & 0.28 & $0.87^{* * * *}$ & 0.11 & $0.51^{*}$ & 0.29 \\
\hline
\end{tabular}

${ }^{*} p<0.05 ;{ }^{* *} p<0.01 ;{ }^{* * *} p<0.001$

(c) BKT, at the station level

\begin{tabular}{cccccccccccc}
\hline \multirow{2}{*}{ Month } & N. of & Amplitudes & \multicolumn{2}{c}{ ST19_ED42 } & \multicolumn{2}{c}{ ZA19_ED42 } & \multicolumn{2}{c}{ ST39_ED42 } & \multicolumn{2}{c}{ ZA39_ED42 } \\
\cline { 4 - 11 } & Days & $(\mathrm{ppm})$ & $\mathrm{R}$ & $\mathrm{A}_{\mathrm{m}} / \mathrm{A}_{\mathrm{o}}$ & $\mathrm{R}$ & $\mathrm{A}_{\mathrm{m}} / \mathrm{A}_{\mathrm{o}}$ & $\mathrm{R}$ & $\mathrm{A}_{\mathrm{m}} / \mathrm{A}_{\mathrm{o}}$ & $\mathrm{R}$ & $\mathrm{A}_{\mathrm{m}} / \mathrm{A}_{\mathrm{o}}$ \\
\hline 201301 & 27 & 23.1 & 0.04 & 0.07 & $0.52^{* *}$ & 0.08 & -0.07 & 0.05 & $0.52^{* *}$ & 0.11 \\
201302 & 17 & 20.3 & 0.09 & 0.07 & $0.54^{* *}$ & 0.06 & -0.25 & 0.04 & $0.66^{* * *}$ & 0.09 \\
201303 & 23 & 25.3 & -0.08 & 0.06 & $0.46^{*}$ & 0.08 & -0.34 & 0.04 & $0.60^{* *}$ & 0.10 \\
201304 & 20 & 22.3 & -0.17 & 0.06 & 0.26 & 0.06 & $-0.42^{*}$ & 0.04 & $0.48^{*}$ & 0.07
\end{tabular}




\begin{tabular}{ccccccccccc}
201305 & 17 & 19.5 & -0.35 & 0.05 & 0.32 & 0.05 & $-0.64^{* *}$ & 0.05 & $0.50^{*}$ & 0.08 \\
201306 & 18 & 21.3 & $-0.53^{* *}$ & 0.07 & 0.16 & 0.06 & $-0.83^{* * *}$ & 0.06 & 0.40 & 0.08 \\
201307 & 20 & 19.7 & -0.09 & 0.07 & $0.60^{* *}$ & 0.06 & -0.38 & 0.05 & $0.68^{* * * *}$ & 0.07 \\
201308 & 19 & 22.3 & $0.62^{* *}$ & 0.06 & $0.96^{* * *}$ & 0.05 & $0.49^{* *}$ & 0.05 & $0.95^{* * *}$ & 0.07 \\
201309 & 8 & 14.2 & $0.82^{* * *}$ & 0.06 & $0.84^{* * *}$ & 0.11 & $0.71^{* * *}$ & 0.05 & $0.84^{* * *}$ & 0.14 \\
201310 & 16 & 23.8 & -0.09 & 0.06 & 0.40 & 0.06 & -0.37 & 0.04 & $0.61^{* *}$ & 0.07 \\
201311 & 21 & 42.4 & -0.29 & 0.03 & 0.11 & 0.03 & -0.58 & 0.02 & $0.42^{*}$ & 0.04 \\
201312 & 22 & 27.9 & 0.00 & 0.05 & $0.49^{*}$ & 0.06 & -0.22 & 0.03 & 0.37 & 0.08 \\
\hline
\end{tabular}

$45 \quad{ }^{*} p<0.05 ;{ }^{* *} p<0.01 ;{ }^{* * *} p<0.001$

46 (d) BKT, at the surface model level (Level=1)

\begin{tabular}{|c|c|c|c|c|c|c|c|c|c|c|}
\hline \multirow{2}{*}{ Month } & \multirow{2}{*}{$\begin{array}{l}\text { N. of } \\
\text { Days }\end{array}$} & \multirow{2}{*}{$\begin{array}{l}\text { Amplitudes } \\
\text { (ppm) }\end{array}$} & \multicolumn{2}{|c|}{ ST19_ED42 } & \multicolumn{2}{|c|}{ ZA19_ED42 } & \multicolumn{2}{|c|}{ ST39_ED42 } & \multicolumn{2}{|c|}{ ZA39_ED42 } \\
\hline & & & $\mathrm{R}$ & $\mathrm{A}_{\mathrm{m}} / \mathrm{A}_{\mathrm{o}}$ & $\mathrm{R}$ & $\mathrm{A}_{\mathrm{m}} / \mathrm{A}_{\mathrm{o}}$ & $\mathrm{R}$ & $\mathrm{A}_{\mathrm{m}} / \mathrm{A}_{\mathrm{o}}$ & $\mathrm{R}$ & $\mathrm{A}_{\mathrm{m}} / \mathrm{A}_{\mathrm{o}}$ \\
\hline 201301 & 27 & 23.1 & $0.95^{* * *}$ & 0.42 & $0.97^{* * *}$ & 0.48 & $0.96^{* * * *}$ & 0.52 & $0.97^{* * * *}$ & 0.57 \\
\hline 201302 & 17 & 20.3 & $0.96^{* * *}$ & 0.44 & $0.97^{* * * *}$ & 0.50 & $0.97^{* * * *}$ & 0.57 & $0.97^{* * * *}$ & 0.63 \\
\hline 201303 & 23 & 25.3 & $0.97^{* * *}$ & 0.39 & $0.97^{* * * *}$ & 0.46 & $0.96^{* * *}$ & 0.50 & $0.96^{* * * *}$ & 0.54 \\
\hline 201304 & 20 & 22.3 & $0.93^{* * *}$ & 0.40 & $0.96^{* * *}$ & 0.42 & $0.95^{* * * *}$ & 0.51 & $0.96^{* * * *}$ & 0.52 \\
\hline 201305 & 17 & 19.5 & $0.93^{* * * *}$ & 0.47 & $0.96^{* * * *}$ & 0.43 & $0.94^{* * * *}$ & 0.61 & $0.95^{* * * *}$ & 0.59 \\
\hline 201306 & 18 & 21.3 & $0.92^{* * * *}$ & 0.48 & $0.94^{* * * *}$ & 0.40 & $0.93^{* * *}$ & 0.55 & $0.94^{* * * *}$ & 0.51 \\
\hline 201307 & 20 & 19.7 & $0.94^{* * * *}$ & 0.39 & $0.97^{* * * *}$ & 0.37 & $0.96^{* * * *}$ & 0.50 & $0.96^{* * * *}$ & 0.49 \\
\hline 201308 & 19 & 22.3 & $0.92^{* * * *}$ & 0.17 & $0.95^{* * * *}$ & 0.25 & $0.90^{* * * *}$ & 0.25 & $0.94^{* * * *}$ & 0.33 \\
\hline 201309 & 8 & 14.2 & $0.93^{* * *}$ & 0.45 & $0.89^{* * * *}$ & 0.33 & $0.95^{* * * *}$ & 0.52 & $0.88^{* * * *}$ & 0.41 \\
\hline 201310 & 16 & 23.8 & $0.98^{* * * *}$ & 0.42 & $0.97^{* * * *}$ & 0.48 & $0.97^{* * * *}$ & 0.55 & $0.95^{* * * *}$ & 0.61 \\
\hline 201311 & 21 & 42.4 & $0.96^{* * *}$ & 0.21 & $0.97^{* * *}$ & 0.24 & $0.96^{* * *}$ & 0.28 & $0.97^{* * * *}$ & 0.28 \\
\hline 201312 & 22 & 27.9 & $0.86^{* * * *}$ & 0.36 & $0.90^{* * * *}$ & 0.42 & $0.91^{* * * *}$ & 0.45 & $0.92^{* * * *}$ & 0.52 \\
\hline
\end{tabular}

47

${ }^{*} p<0.05 ;{ }^{* *} p<0.01 ;{ }^{* * *} p<0.001$

48 
49 Figure S1 (a) Map of locations of airports in South and East Asia from the Comprehensive 50 Observation Network for TRace gases by AIrLiner (CONTRAIL) project (Machida et al., 51 2008). (b) Close-up map for airports in Japan and Republic of Korea. The whole region is 52 divided into four subregions, namely East Asia (EAS), the Indian sub-continent (IND), 53 Northern Southeast Asia (NSA) and Southern Southeast Asia (SSA), and all the airports and 54 vertical profiles are grouped into the four subregions accordingly. The zoomed grid of the 55 LMDz-INCA model is also plotted as background.
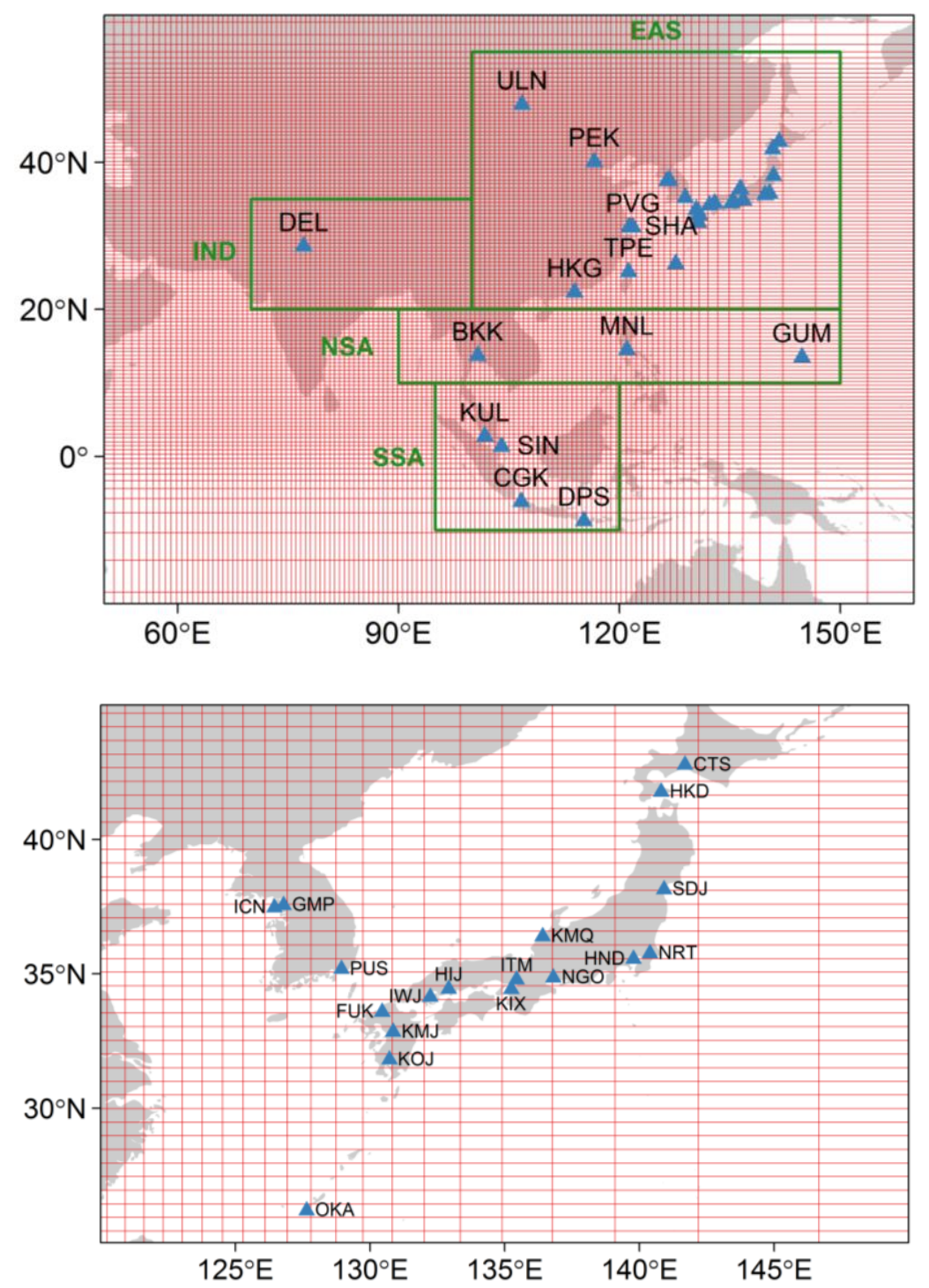
58 Figure S2 Sampling dates of $\mathrm{CO}_{2}$ measurements for airports in Figure S1. For each airport, 59 only sampling dates with vertical profiles available (i.e. measurements during ascending or 60 descending flights) are plotted.

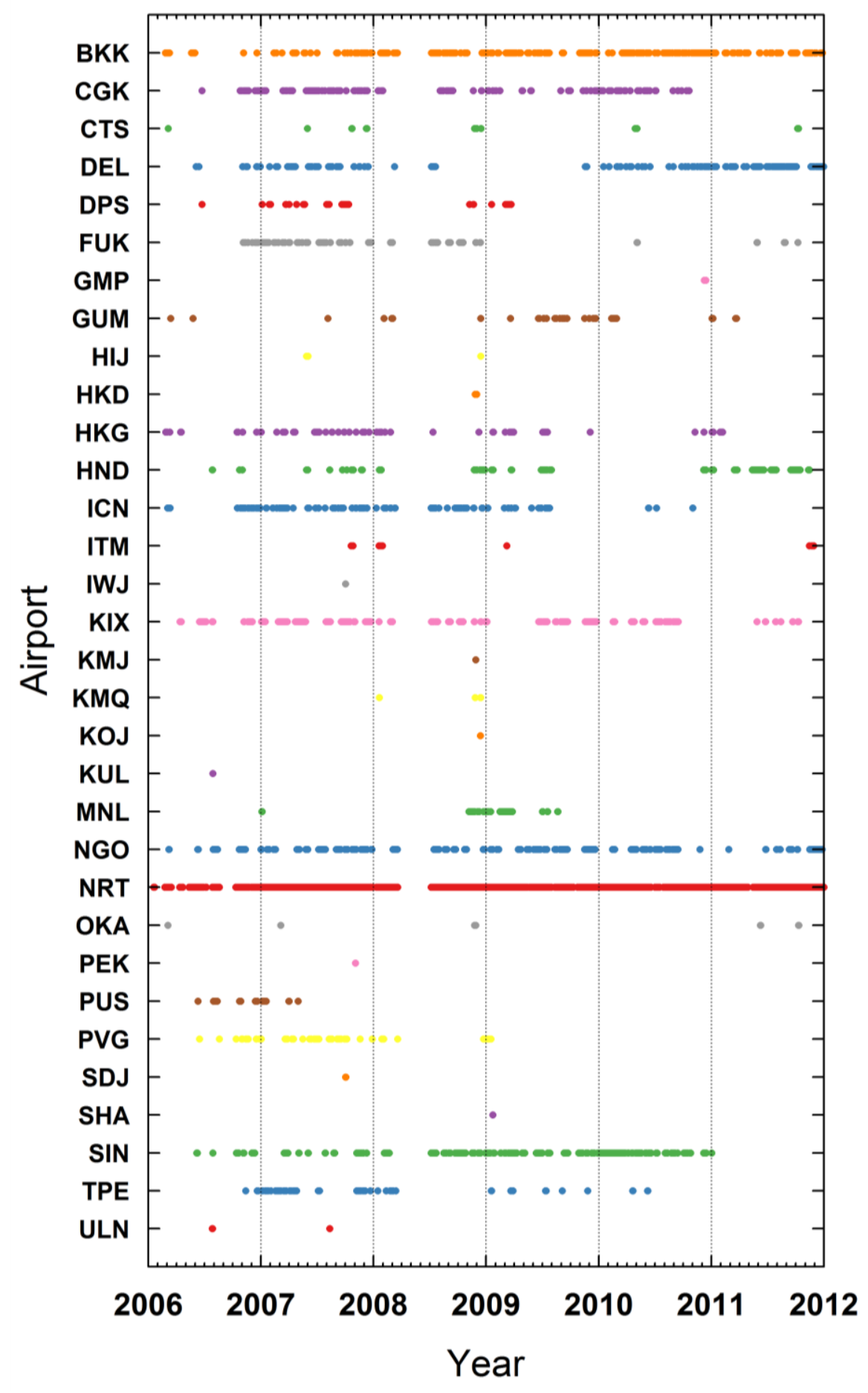


62 Figure $\mathrm{S3}$ Scatterplots of the simulated and observed $\mathrm{CH}_{4}$ mean annual gradients between

63

64

65

66

67

68

69

70

71

(b) April-June

(a) January-March

ST19_ED42 vs ZA19_ED42 ST39_ED42 vs ZA39_ED42

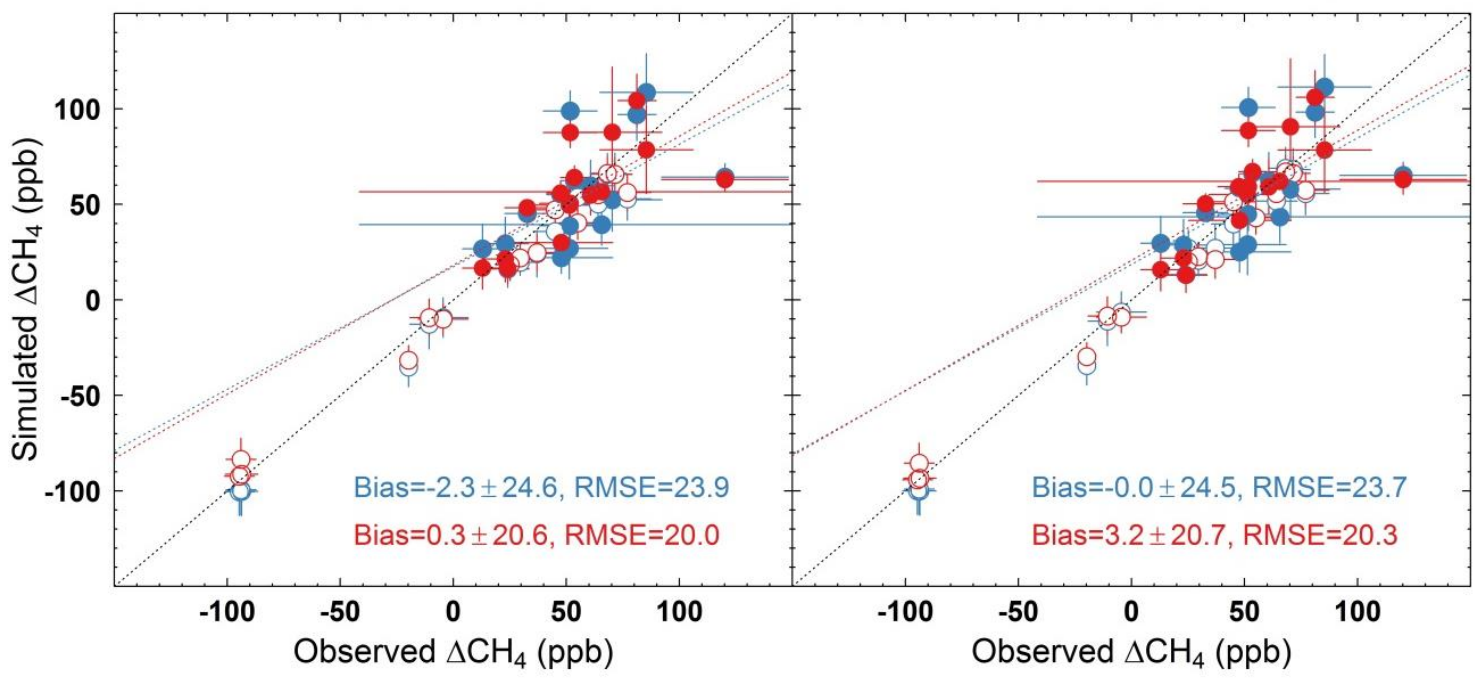

ST19_ED42 vs ZA19_ED42 ST39_ED42 vs ZA39_ED42

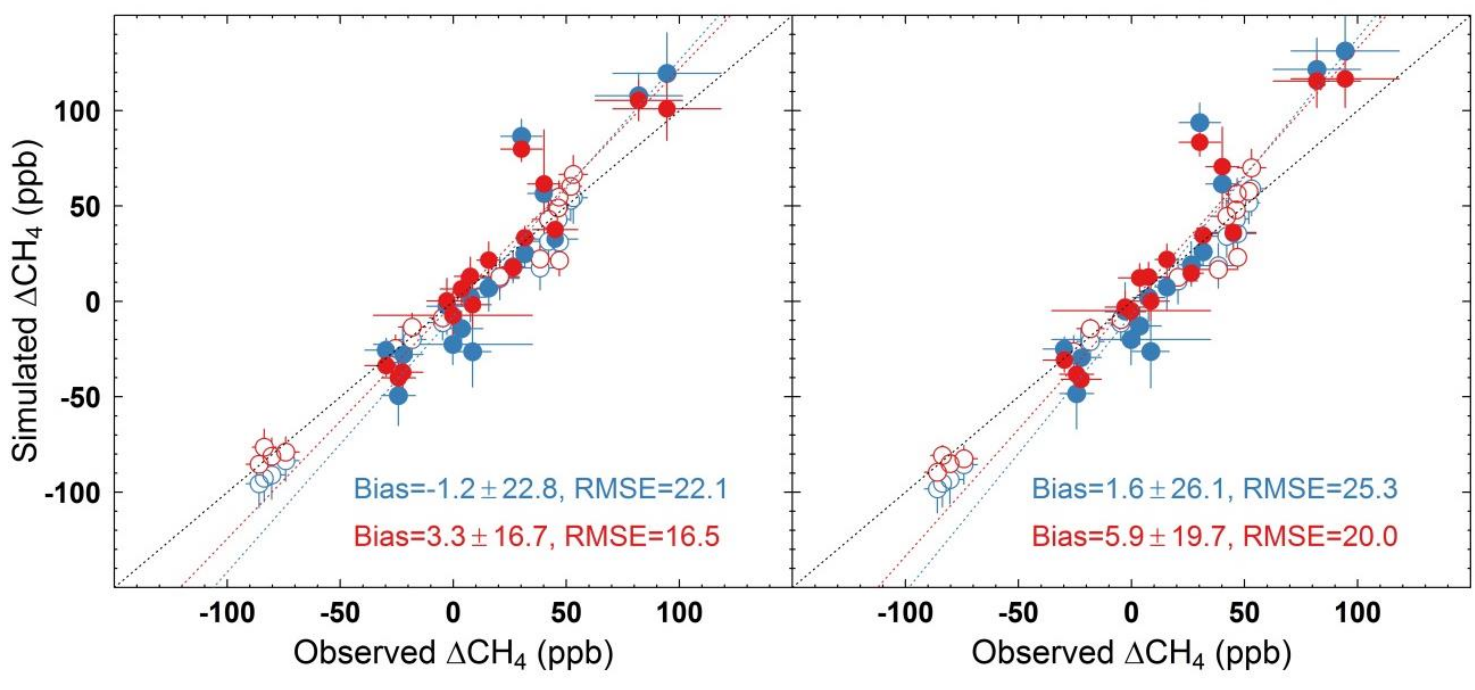




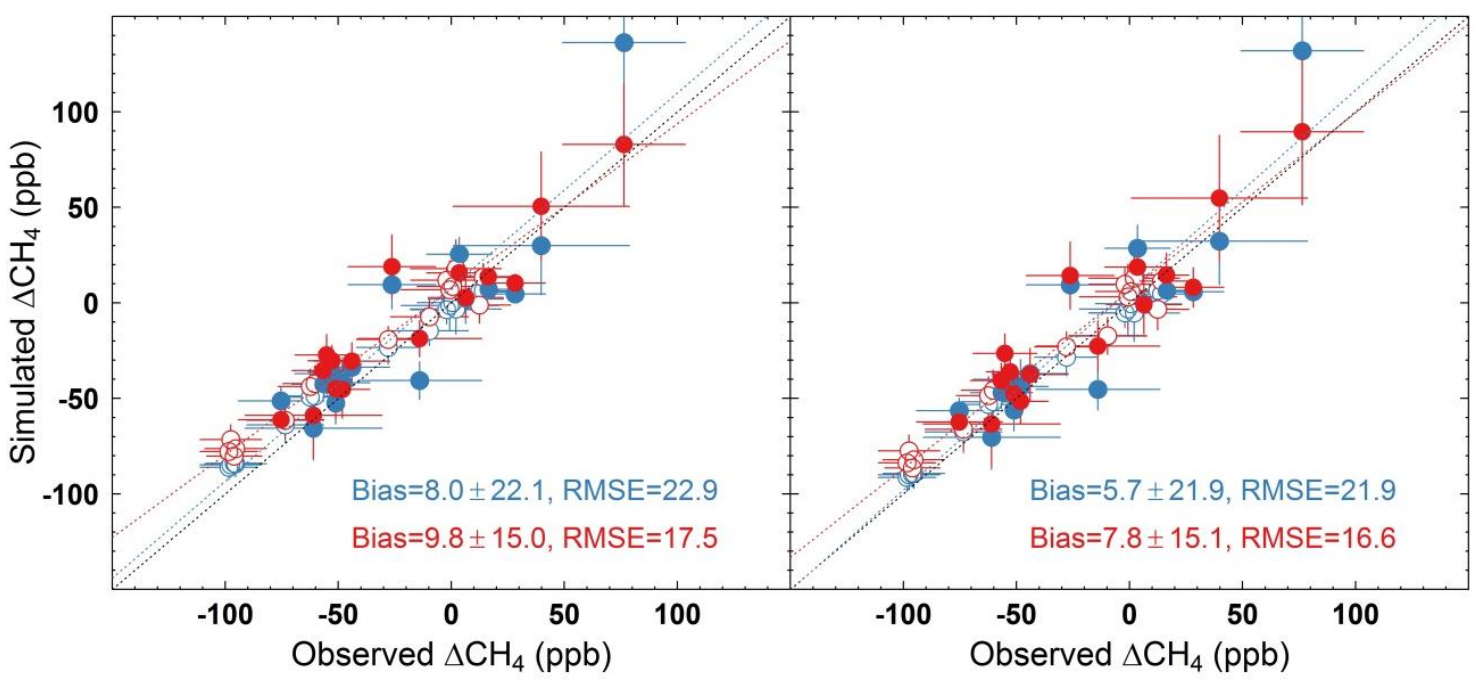

(d) October-December

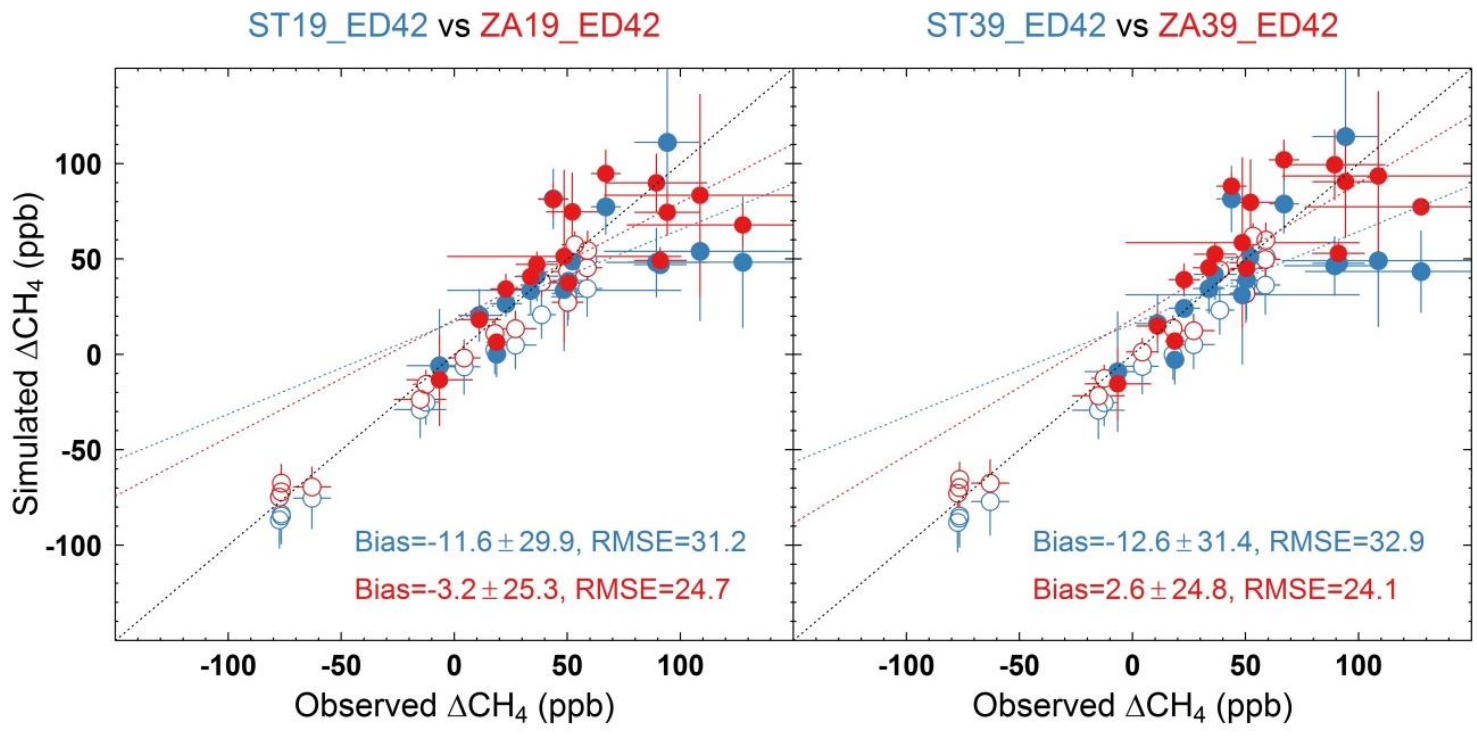


79 Figure S4 $\mathrm{CH}_{4}$ surface flux maps for South and East Asia (SEA), based on two different 80 inventories of anthropogenic emissions for the year 2010 from EDGARv4.2FT2010 and 81 EDGARv4.3.2 (http://edgar.jrc.ec.europa.eu). $\mathrm{CH}_{4}$ hotspots, defined as the grids with 82 emission rates stronger than $1 \times 10^{-9} \mathrm{~kg} \mathrm{CH}_{4} \mathrm{~m}^{-2} \mathrm{~s}^{-1}\left(\approx 0.8 \mathrm{Tg} \mathrm{CH}_{4} \mathrm{yr}^{-1}\right)$, are indicated by blue 83 dots. Both maps are generated in ZA grid meshes and with the same biogenic $\mathrm{CH}_{4}$ fluxes as 84 given in Table 1.

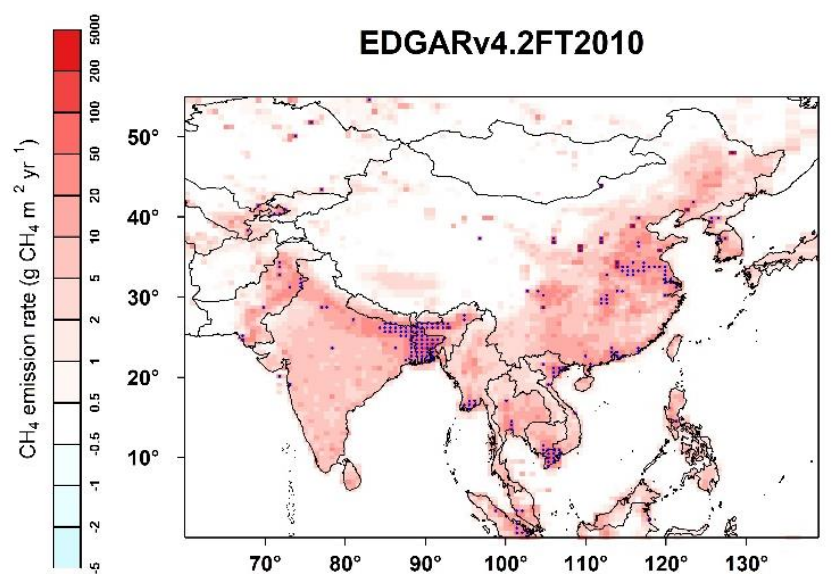

EDGARv4.3.2

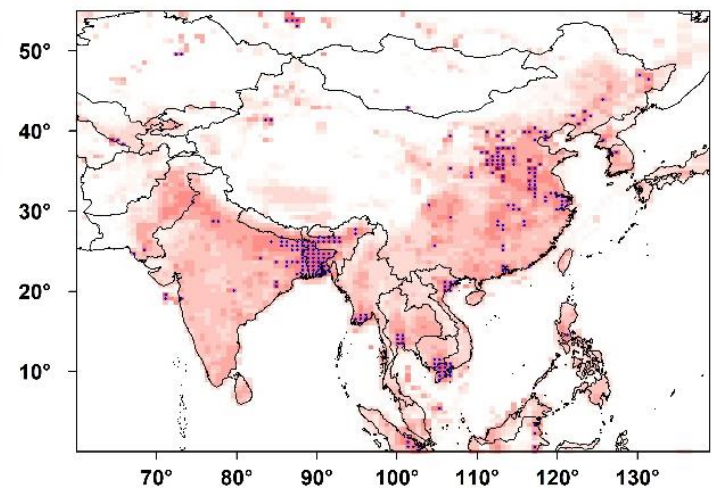


87 Figure S5 Maps of $\mathrm{CH}_{4}$ surface fluxes (upper panels) and $\mathrm{CH}_{4}$ concentration fields at the

88 first model level (lower panels) for the year 2010. Results from both ZA and ST with 19

89 model layers

90 are presented for comparison.
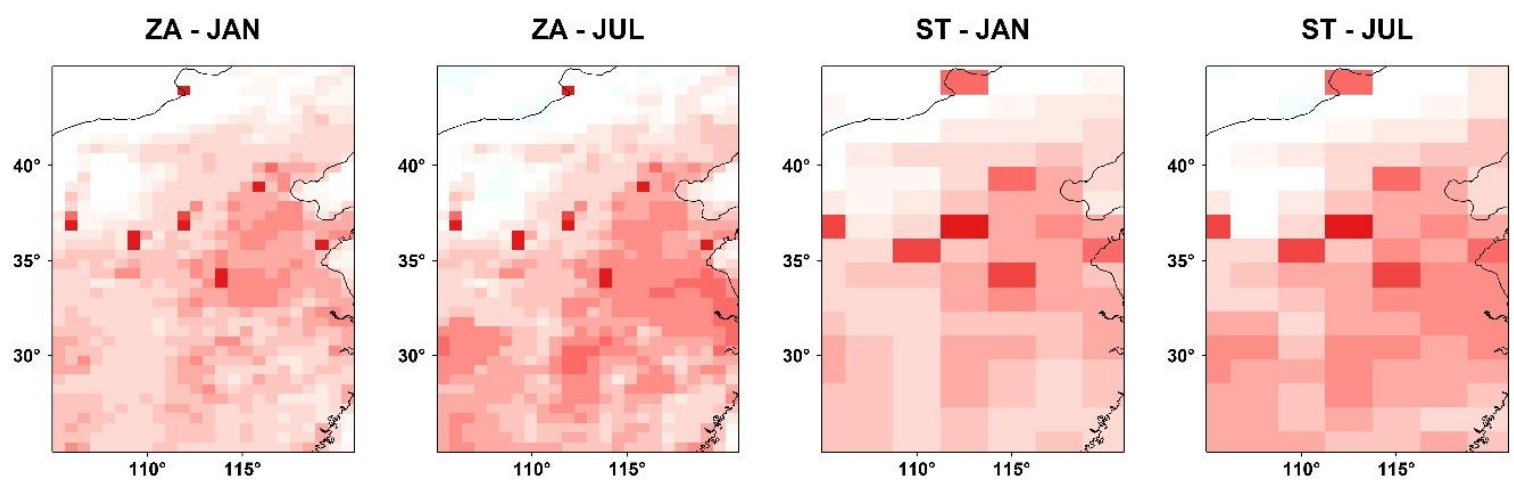

91

$\mathrm{CH}_{4}$ emission rate $\left(\mathrm{g} \mathrm{CH}_{4} \mathrm{~m}^{-2} \mathrm{yr}^{-1}\right)$

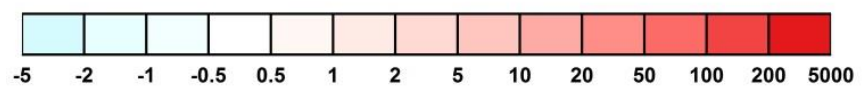

92
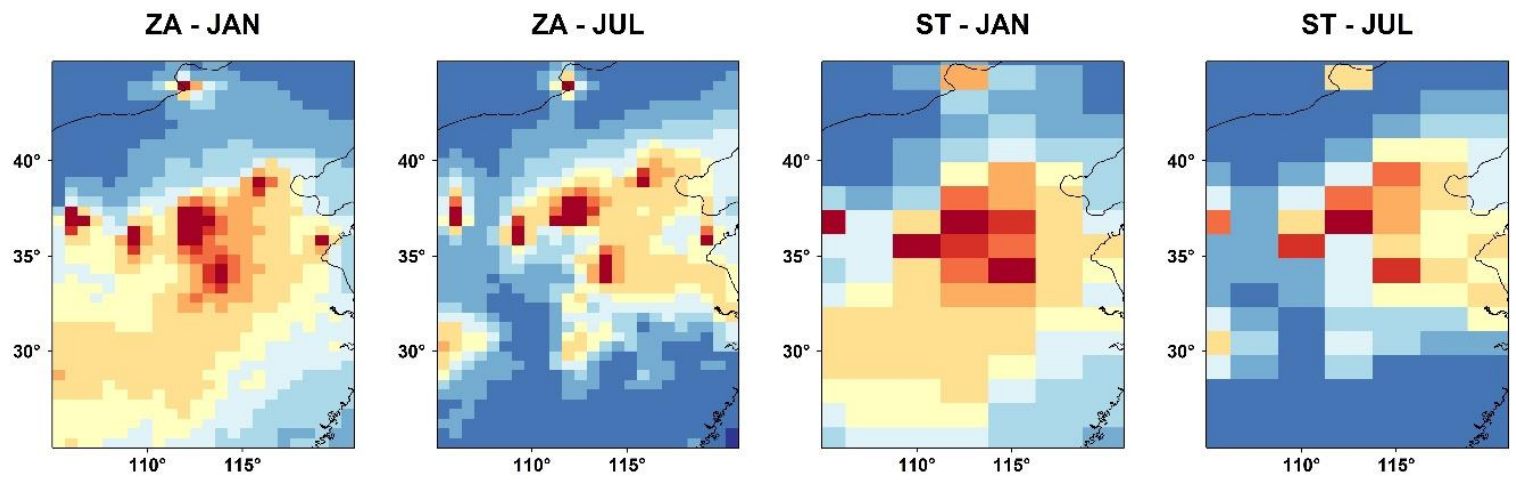

93

$\mathrm{CH}_{4}$ (ppb)

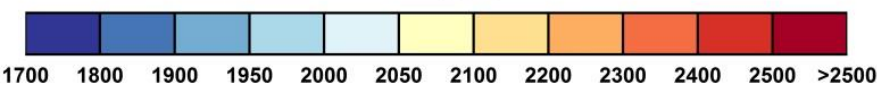


95

96

97

98

99

100
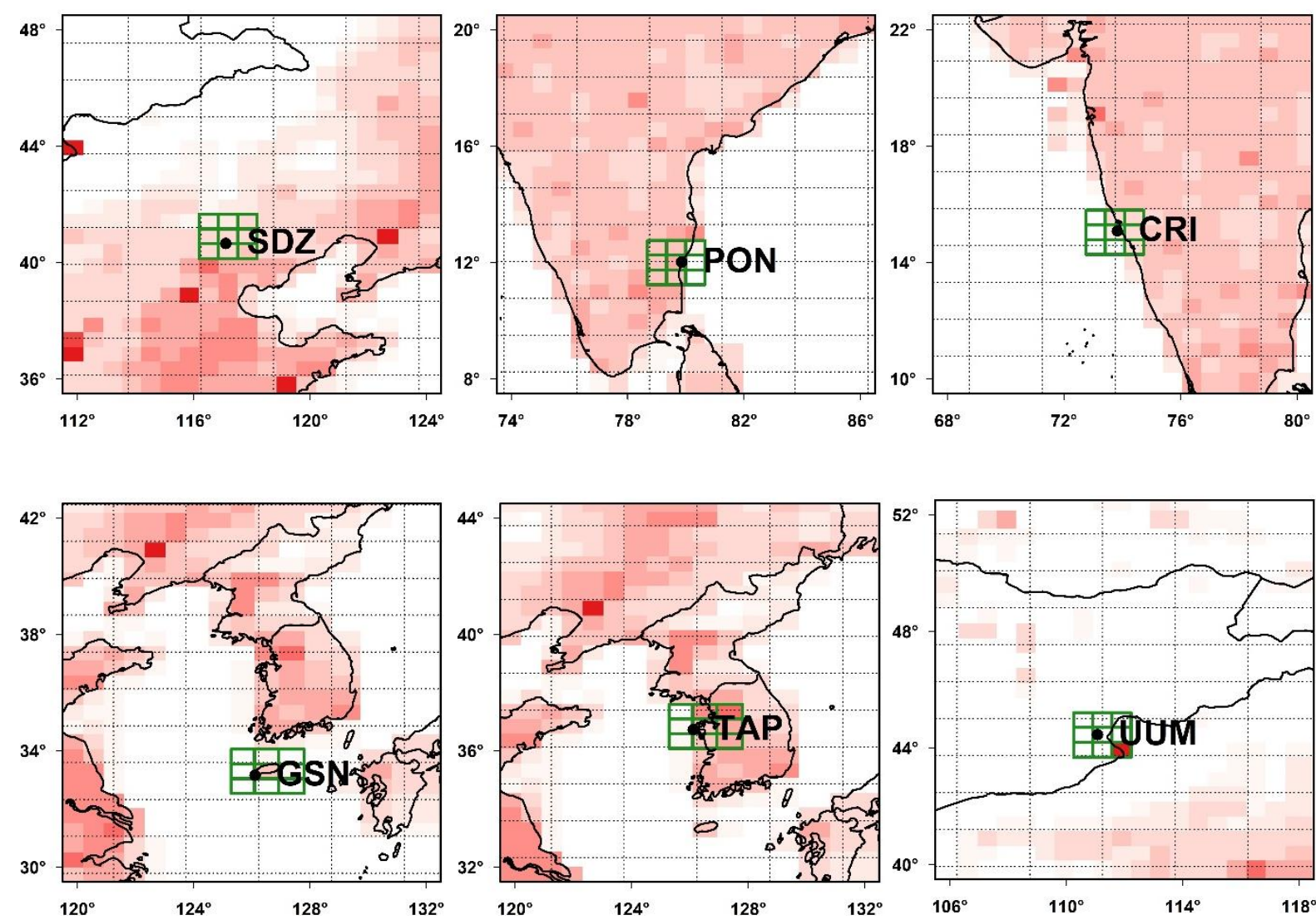

$\mathrm{CH}_{4}$ emission rate $\left(\mathrm{g} \mathrm{CH}_{4} \mathrm{~m}^{-2} \mathrm{yr}^{-1}\right)$

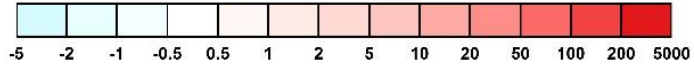


104

105

106

107

108

109

110

111

112

113

Figure S7 Scatterplots of simulated and observed $\mathrm{CO}_{2}$ mean annual gradients between HLE and other stations for January-March (a), April-June (b), July-September (c), and OctoberDecember (d). In each panel, the simulated $\mathrm{CO}_{2}$ gradients are based on simulations from the standard (blue circles) and zoom (red circles) versions, respectively. The black dotted line indicates the identity line, whereas the blue and red dotted lines indicate the corresponding linear fitted lines. The closed and open circles represent stations inside and outside the zoomed region.

(a) January-March

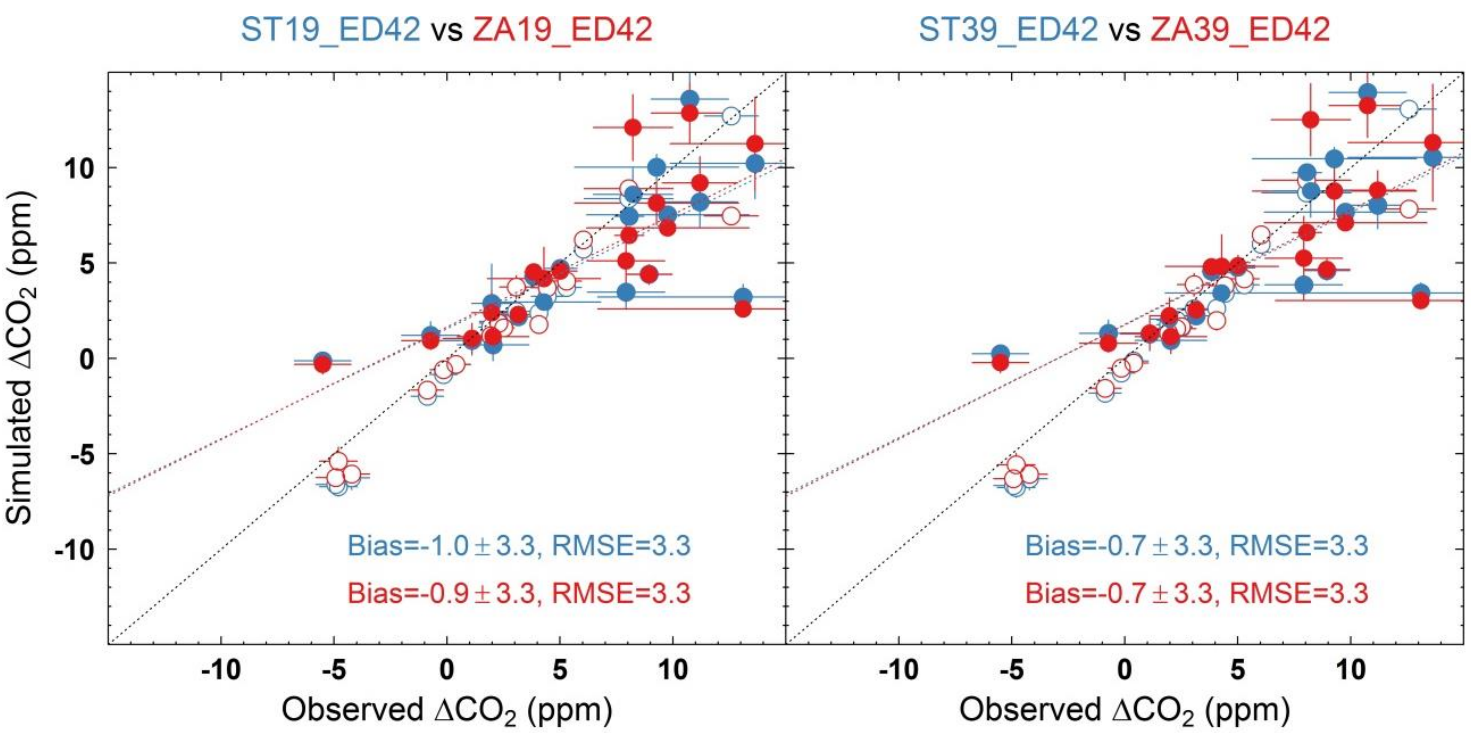

(b) April-June

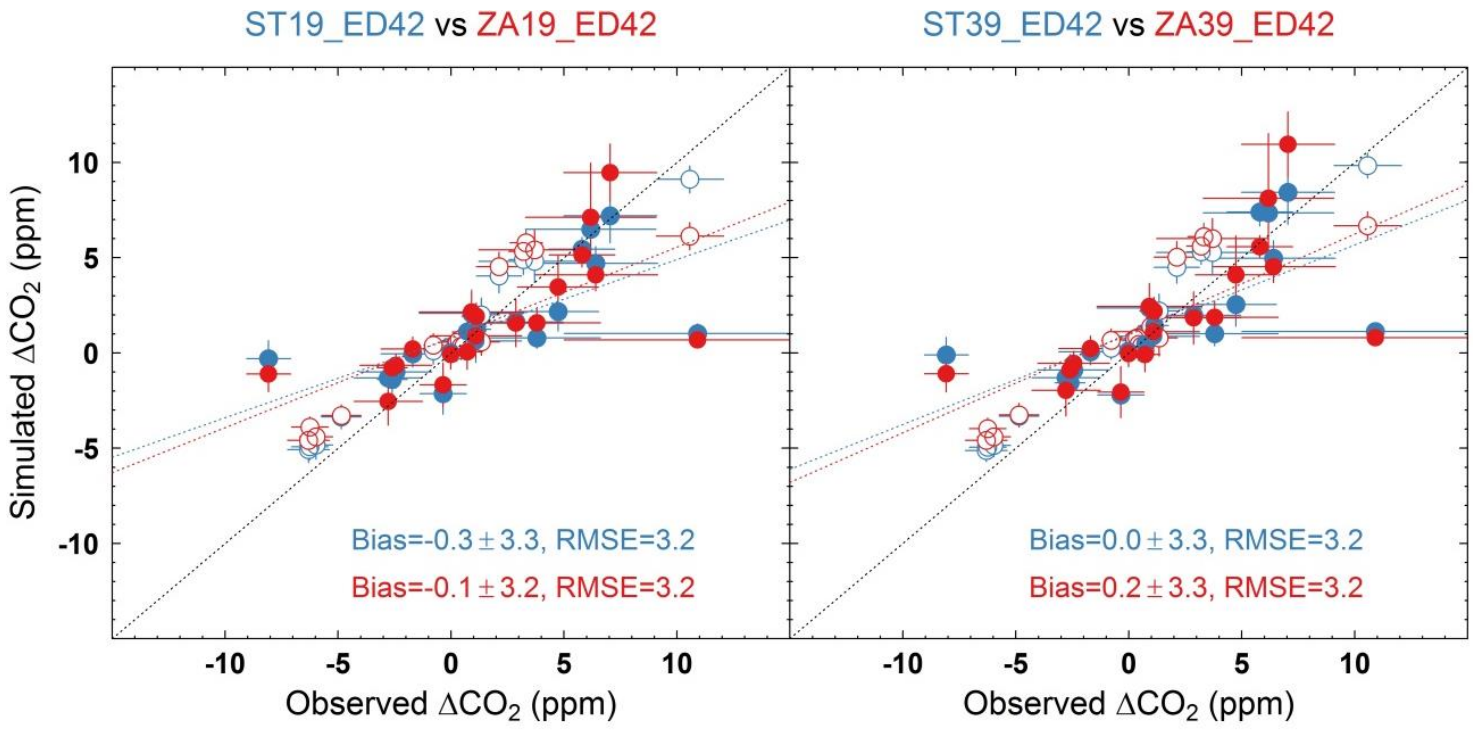




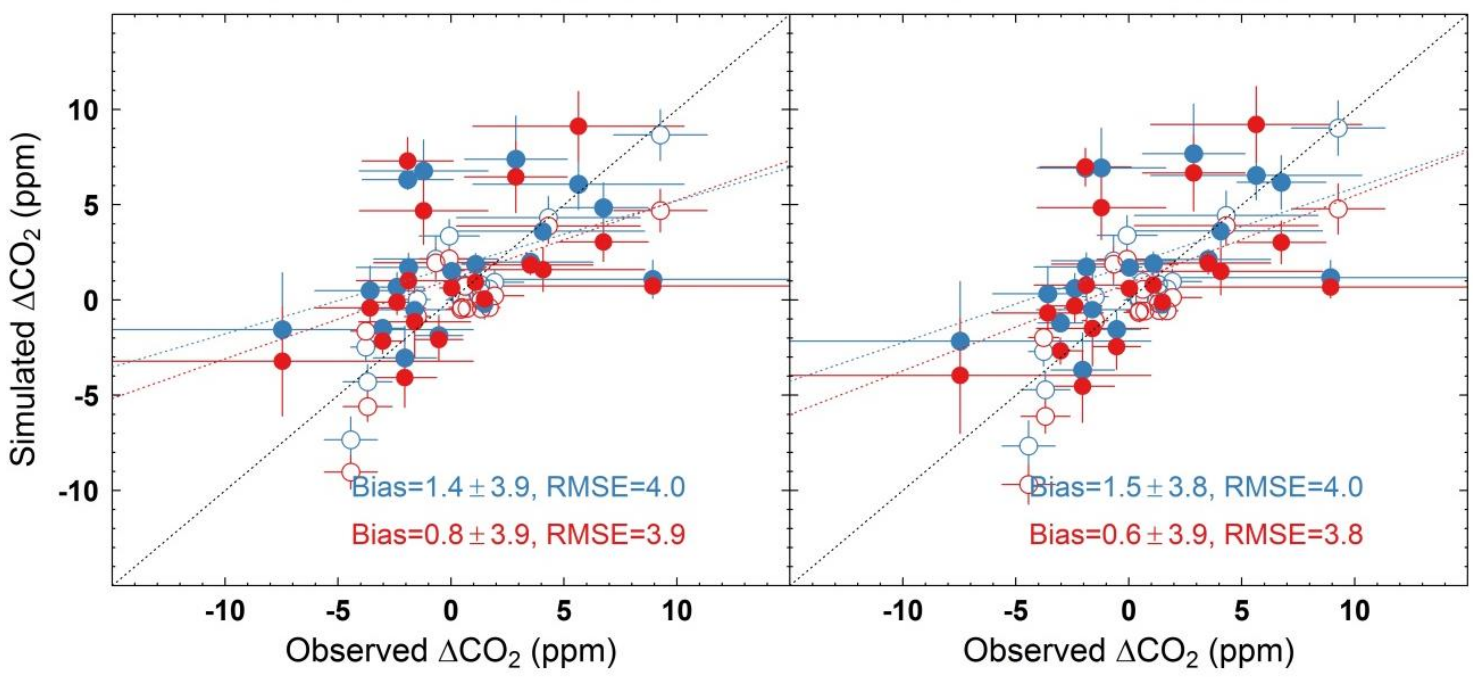

118 (d) October-December

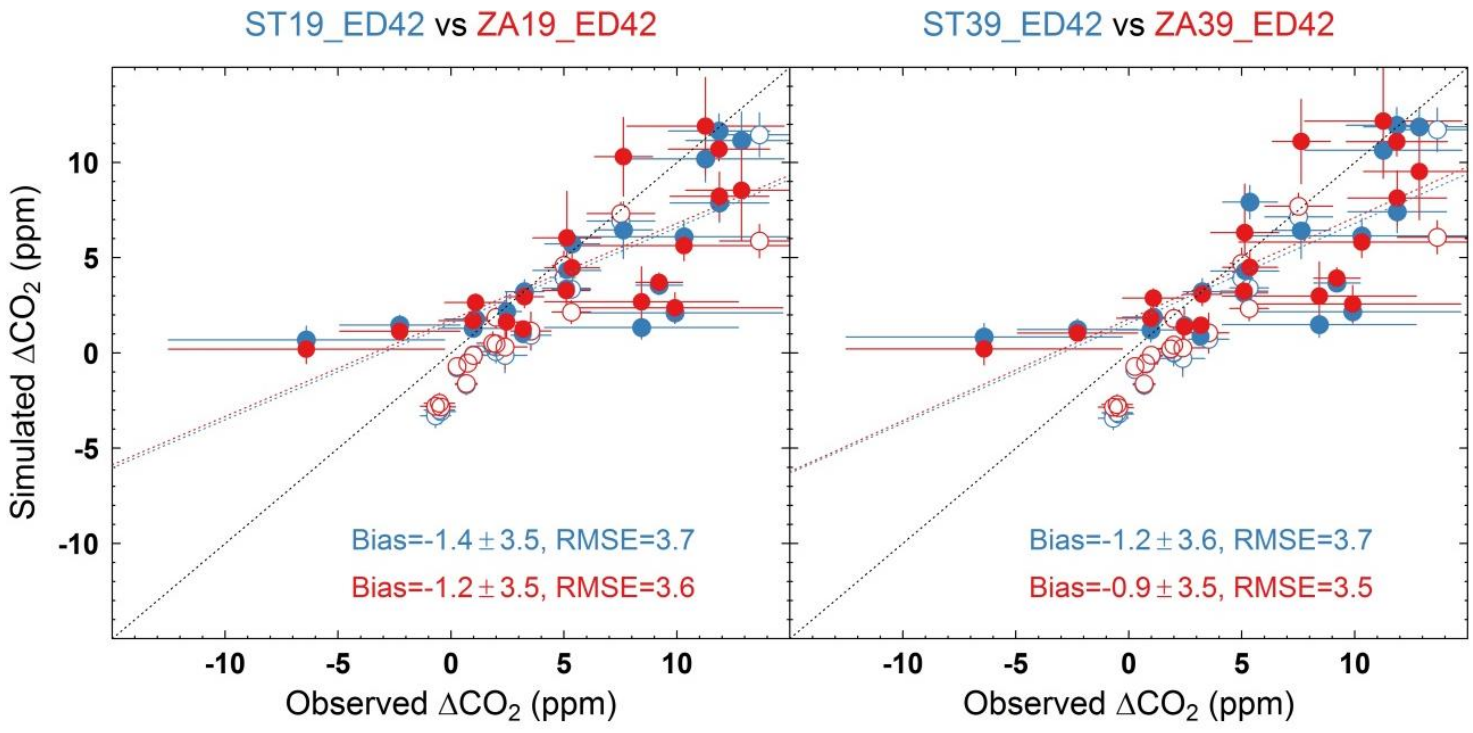


122 Figure $\mathbf{S 8}$ The spatial distributions of mean annual $\mathrm{CO}_{2}$ fluxes around the station TAP for the 123 year 2010 mapped with the ZAs model grids. The black meshes indicate the STs model grids. 124 The black dot denotes the location of the station, whereas the $3 \times 3$ meshes colored in green 125 indicate the grid where the station is located (the 'center grid') and its 8 neighbors.

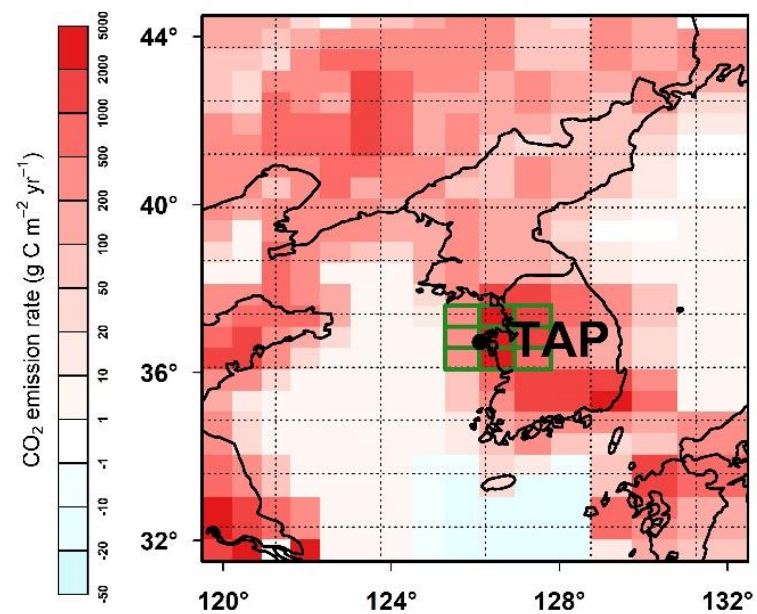


127 Figure S9 The observed and simulated mean seasonal cycles of $\mathrm{CH}_{4}$ for $\mathrm{KZM}$, WLG and 128 UUM. In each panel, the colors of lines are defined as Figure 3. In addition, we also show the 129 mean seasonal cycles from sensitivity test simulations prescribed with wetland emissions 130 from ORCHIDEE outputs (green and orange lines for the standard and zoom versions, 131 respectively). The text shows statistics between the simulated and observed seasonal cycles 132 for 39-layer models.
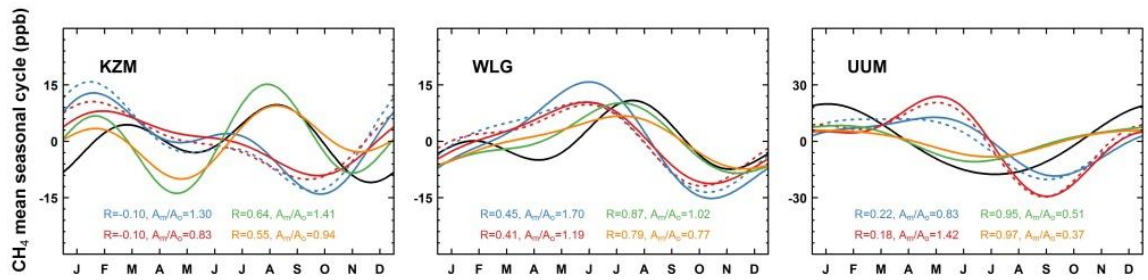
134 Figure S10 The observed and simulated mean seasonal cycles of $\mathrm{CH}_{4}$ for stations outside the 135 zoomed region. In each panel, the simulated mean seasonal cycles are based on simulations 136 from the standard (blue lines) and zoom (red lines) versions, respectively. The text shows 137 statistics between the simulated and observed seasonal cycles for 39-layer models.
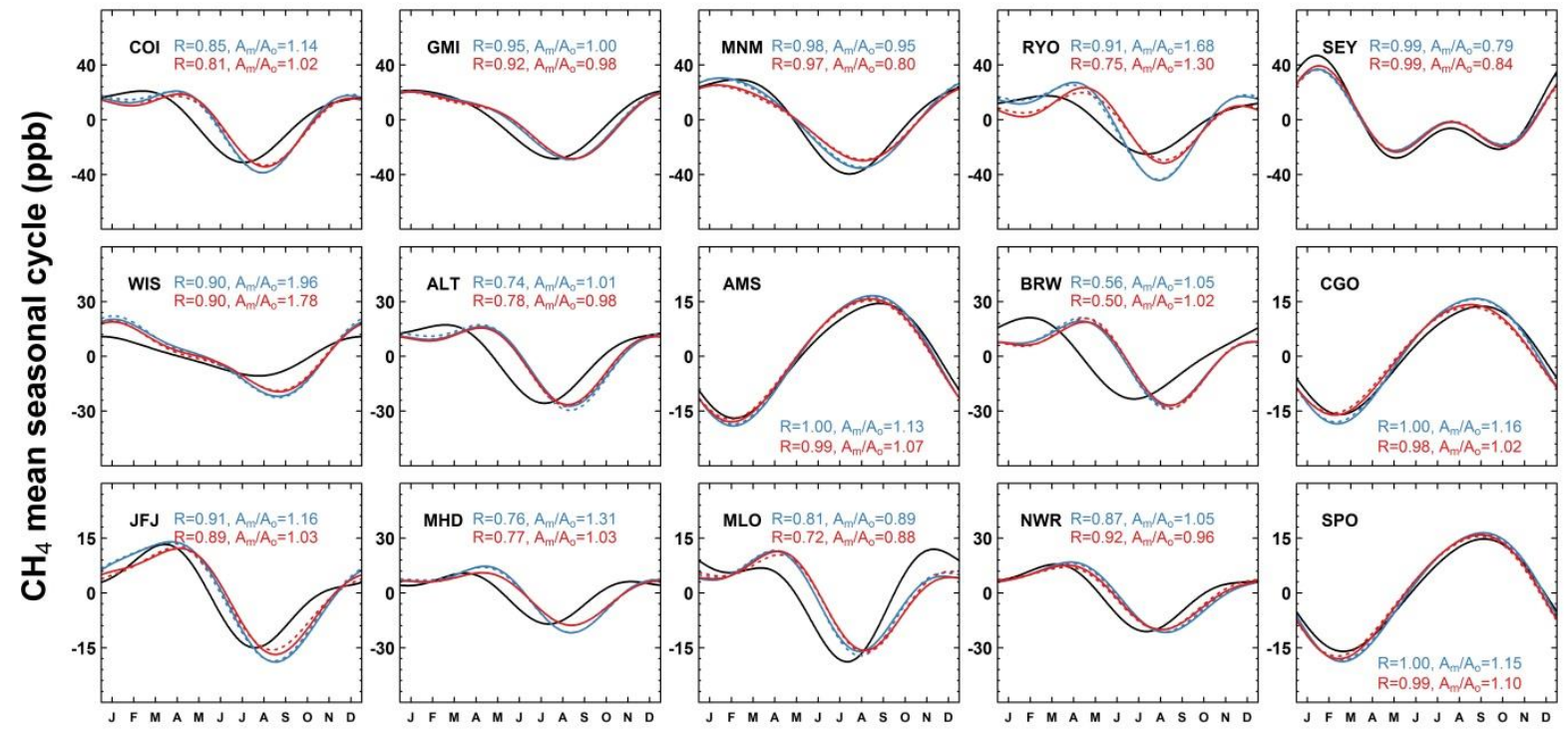
140 Figure S11 The observed and simulated mean seasonal cycles of $\mathrm{CO}_{2}$ for stations outside the 141 zoomed region. In each panel, the simulated mean seasonal cycles are based on simulations 142 from the standard (blue lines) and zoom (red lines) versions, respectively. The text shows 143 statistics between the simulated and observed seasonal cycles for 39-layer models.
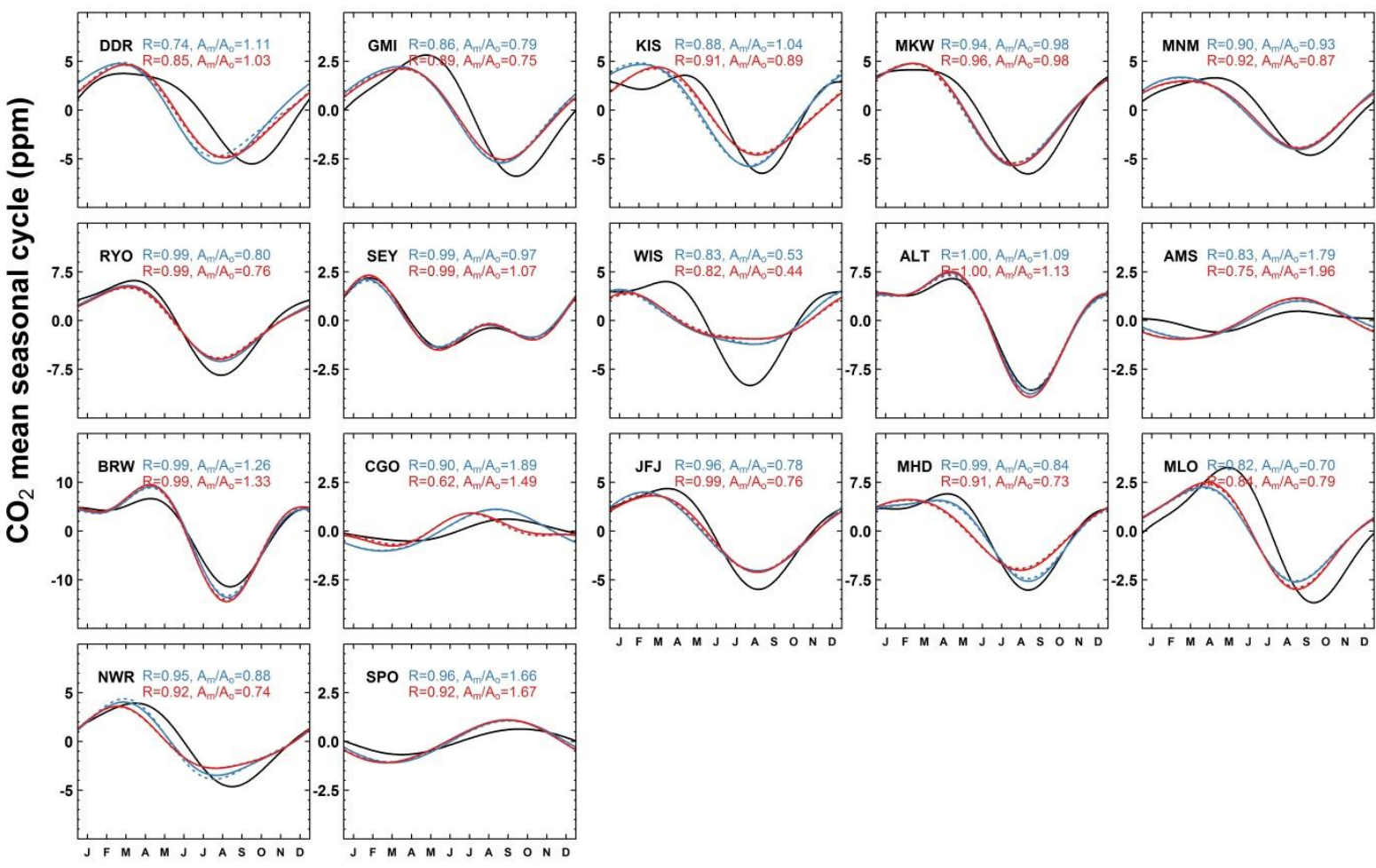

AMS $R=0.83, A_{m} / A_{0}=1.79$
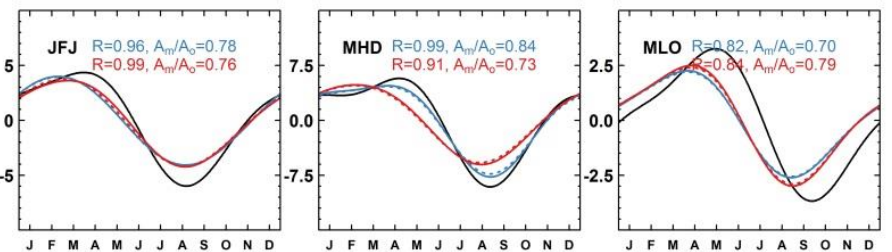
Figure S12 The correlations and normalized standard deviations between the simulated and observed synoptic variability for $\mathrm{CH}_{4}(\mathbf{a}, \mathbf{b})$ and $\mathrm{CO}_{2}(\mathbf{c}, \mathbf{d})$ at stations outside the zoomed region. For each station, the synoptic variability is calculated from residuals from the smoothed fitting curve.

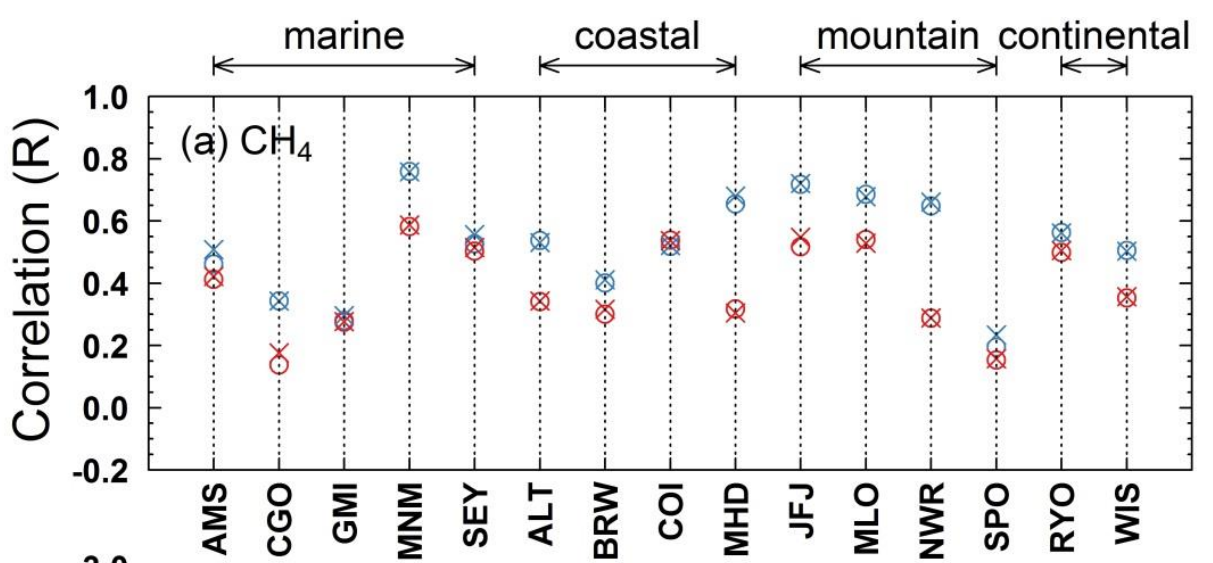

150
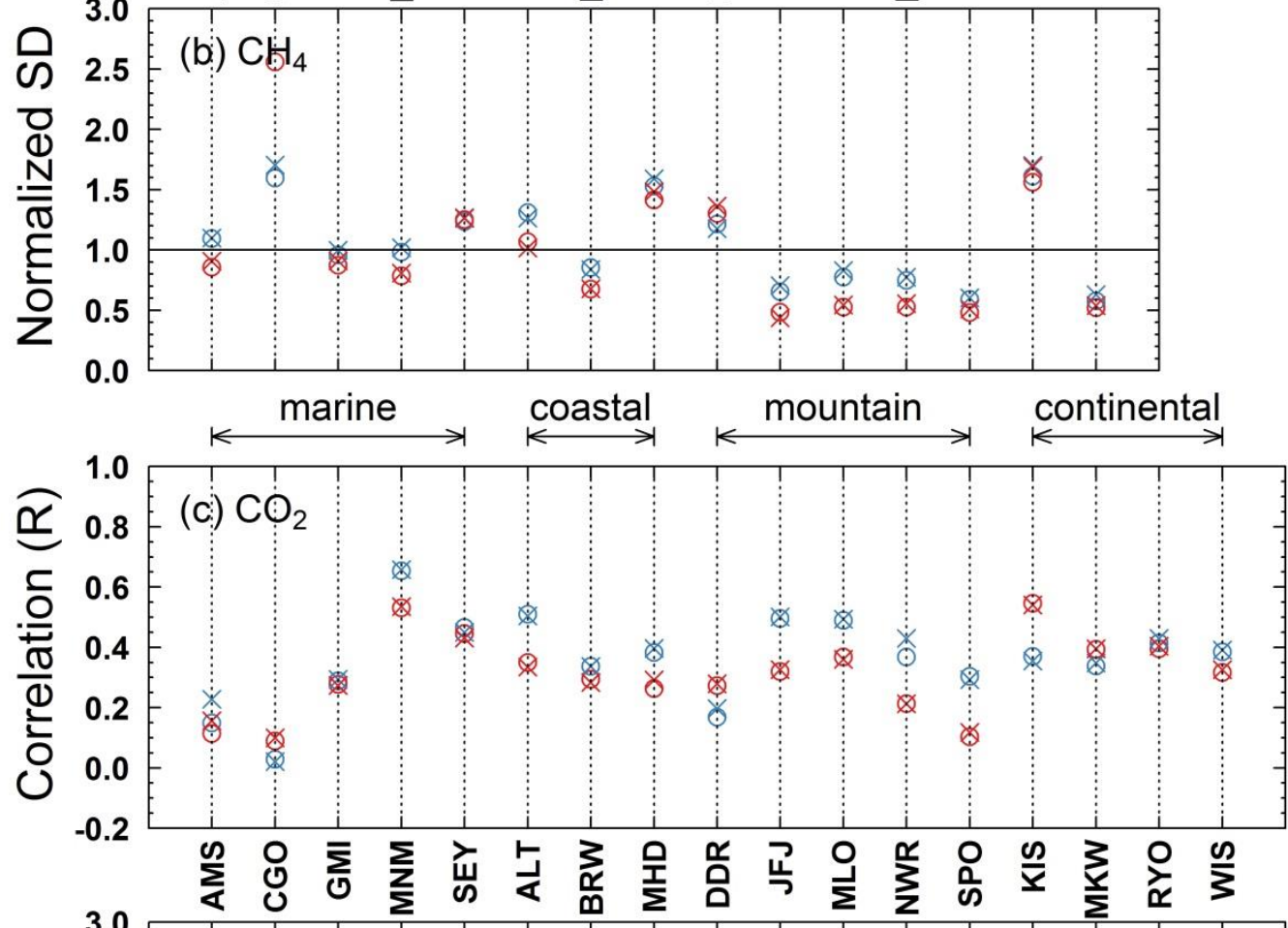

151

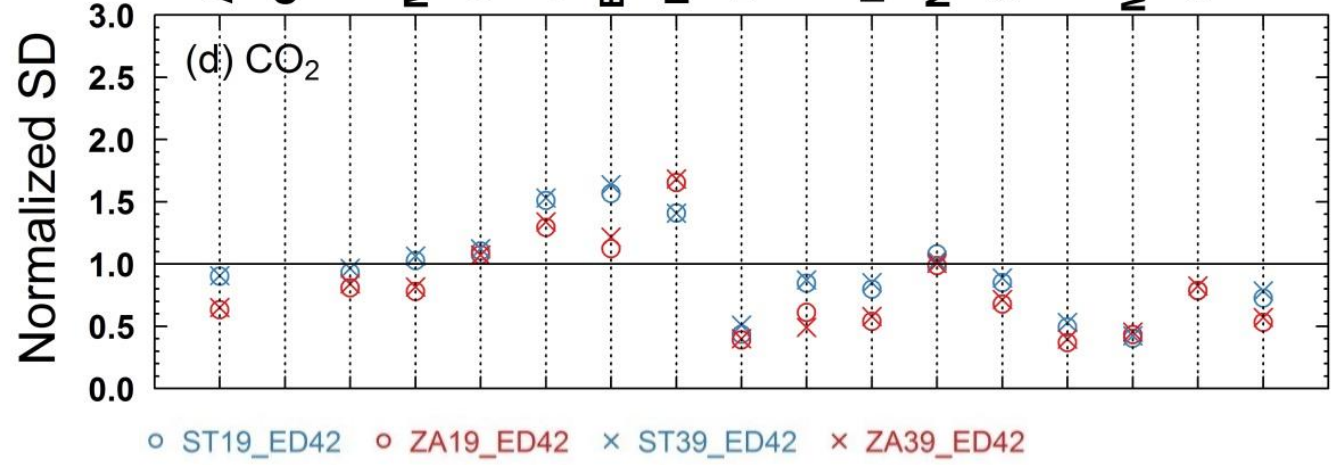


154 Figure S13 Time series of observed and simulated $\mathrm{CH}_{4}$ synoptic variabilities at UUM over 155 the period 2006-2013. The synoptic variability is calculated from residuals from the 156 smoothed fitting curve.

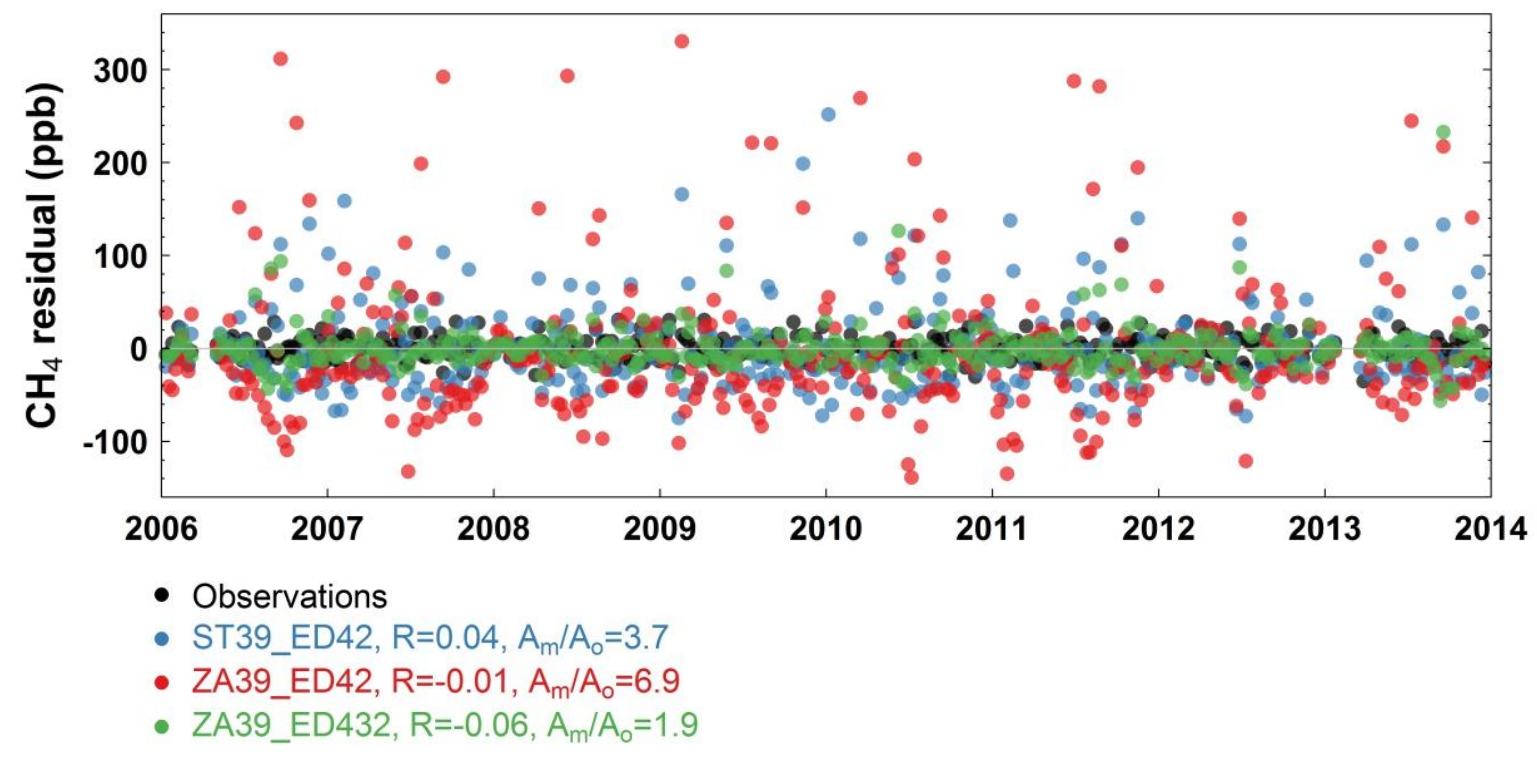

159 
160

161

162

163

164

165

166

167
Figure S14 Time series of observed and simulated $\mathrm{CH}_{4}(\mathbf{a})$ and $\mathrm{CO}_{2}(\mathbf{b})$ synoptic variabilities at PON over the period 2006-2013. For each trace gas, the synoptic variability is calculated from residuals from the smoothed fitting curve.
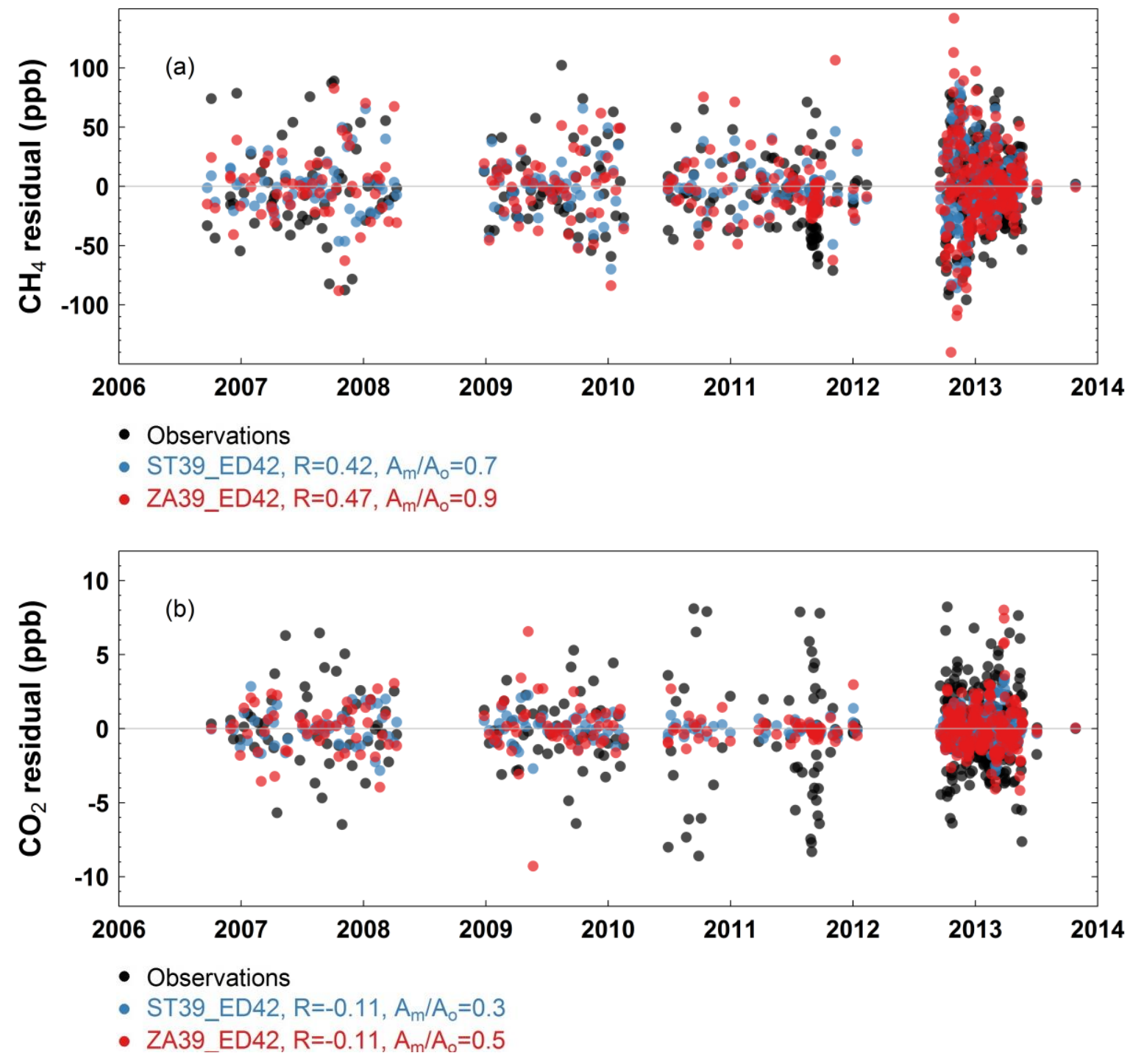
168 Reference

169 Machida, T., Matsueda, H., Sawa, Y., Nakagawa, Y., Hirotani, K., Kondo, N., Goto, K.,

170 Nakazawa, T., Ishikawa, K. and Ogawa, T.: Worldwide Measurements of Atmospheric $\mathrm{CO}_{2}$

171 and Other Trace Gas Species Using Commercial Airlines, J. Atmos. Ocean. Technol., 25(10), 172 1744-1754, doi:10.1175/2008JTECHA1082.1, 2008. 